\title{
4. Der positivistische Objektivismus
}

Nachdem die postmodern-narrativistischen Einwände gegen historische Objektivität abgewiesen worden sind und ein minimalistisches Narrativitätsmodell entworfen worden ist, stellt sich die Aufgabe, die Vereinbarkeit von Objektivität (i. S. des Komponentenmodells) mit Standortgebundenheit gegen eine theoretische Position zu verteidigen, die Objektivität besonders rigide interpretiert. Diese Position soll als positivistischer Objektivismus $^{192}$ bezeichnet werden. Sie ist eine Extremversion des Objektivismus, die Kutschera als ,falsche Objektivität“ bezeichnet und die in der philosophischen Literatur etwa von Nagel und Rorty kritisiert worden ist. ${ }^{193}$

Das Kernelement der „falschen Objektivität“" ist die Vorstellung einer Erkenntnis der Objekte in der Welt ,wie sie an sich sind“, das bedeutet, wie sie restlos unabhängig von den ,Verzerrungen' durch eine Perspektive er-

192 Mit der Bezeichnung ,positivistisch“ soll keineswegs gesagt werden, dass alle Theoretiker, die dem Positivismus zugerechnet werden, genau diese Auffassung von Objektivität vertreten haben oder vertreten müssten. Darüber hinaus muss an dieser Stelle betont werden, dass der ,positivistische Objektivismus“ hier nicht anhand eines Autors oder einer ganz bestimmten Theorieschule diskutiert werden soll. Es handelt sich um eine denkbare, systematische Gegenposition, die idealtypisch aus einer ganzen Reihe von Aussagen unterschiedlichster Provenienz zusammengestellt worden ist. Man könnte sie historisch am ehesten einem kruden Historismus annähern. Sie kann jedoch nicht kurzerhand mit dem Historismus schlechthin gleichgesetzt werden, wie das gelegentlich geschieht. Mit einer solchen Identifikation wird nur eine der vielen Begriffsdifferenzierungen des Historismus eingefangen. Vgl. Wittkau 1994 für einen Überblick über die Vielgestaltigkeit des Historismus. Systematisch wird diese Position von Munslow 2003 (und 1997) unter dem Begriff des „Rekonstruktionismus“ diskutiert. Kutschera 1993 baut in einem ähnlichen idealtypischen Manöver eine analoge Gegenposition, die er „Materialismus“ nennt, zu seiner Theorie auf. Appleby et al. 1994 diskutieren diese Position unter dem Titel ,heroic model of science“, das sie historisch bis zur Aufklärung zurückverfolgen (ebd., 15-51).

193 Kutschera 1993, 1 und Kap. 9; Nagel 1991 und 1986; Rorty 1991. 
kannt werden können. Paradigmatisch für diese Form des Objektivismus sei, laut Kutschera, der Physikalismus, der jede Erkenntnis auf eine von aller sinnlichen Anschauung und menschlichen Perspektive bereinigte Physik reduzieren möchte, um auf diese Weise zu erkennen, wie die Dinge an sich sind. Der Fokus liegt auf der unverzerrten Korrespondenz zwischen dem Erkannten (,der Welt') und unserer Erkenntnis bzw. unserer Darstellung des Erkannten. Damit ist der korrespondenztheoretische Kern von Objektivität in extremis zu einem epistemologischen Absolutismus erhoben worden, der Objektivität mit dem Zugang zu einer unverzerrten Abbildung der Welt identifiziert; der also ganz im Geist der Realdefinitionsstrategie die konzeptuelle Vielfalt des Objektivitätsbegriffs auf eine einzige Spielart, in diesem Fall die maximal mögliche Neutralität des Historikers, sprich, die Auslöschung seiner Persönlichkeit reduziert. Mit dieser Idealvorstellung von Erkenntnis geht eine ganze Reihe von weiteren epistemologisch und wissenschaftstheoretischen Grundüberzeugungen einher, die eine solche Idealvorstellung überhaupt erst machbar erscheinen lassen. Sie gilt es im folgenden herauszuarbeiten und knapp zu skizzieren. 


\subsection{Grundzüge des positivistischen Objektivismus}

Zentrale Züge des positivistischen Objektivismus sind bereits 1935 von Charles Beard in seiner Auseinandersetzung mit seinem Kollegen T. C. Smith herausgestellt worden:

Can Mr. Smith's noble dream, his splendid hope, be realized in fact? [...] This theory that history as it actually was can be disclosed by critical study, can be known as objective truth, and can be stated as such, contains certain elements and assumptions. The first is that history [...] has existed as an object or series of objects outside the mind of the historian (a Gegenüber separated from him and changing in time). The second is that the historian can face and know this object or series of objects and can describe it as it objectively existed. The third is that the historian can [...] divest himself of all taint of religious, political, philosophical, social, sex, economic, moral, and aesthetic interests, and view his Gegenüber with strict impartiality somewhat as the mirror reflects any object to which it is held up. The fourth is that the multitudinous events of history as actuality have some structural organization through inner (perhaps causal) relations, which the impartial historian can grasp by inquiry and observation and accurately reproduce or describe in written history. The fifth is that the substances of this history can be grasped in themselves by purely rational or intellectual efforts [...]. ${ }^{194}$

Der positivistische Objektivismus vertritt demnach eine Reihe von epistemologisch-wissenschaftstheoretischen Grundüberzeugungen: Zunächst handelt es sich um die Grundüberzeugung, dass Objektivität dann erreicht ist, wenn eine korrespondierende Darstellung der Sachverhalte, die in der Welt bestehen, gegeben worden ist. Dabei kann Korrespondenz nur vorliegen, wenn potentielle Verzerrungen ausgeschaltet worden sind. Der positivistische Objektivist ist, will er dieses Ziel erreichen, dazu gezwungen, eine monistische Sicht auf narrative Redeskriptionen einzunehmen, die da-

194 Beard 1964, 317. 
von ausgeht, dass eine Pluralität von Darstellungen nur insofern gerechtfertigt werden kann, als die narrative Pluralität eine leider zu durchlaufende und $\mathrm{zu}$ überwindende Durchgangsstation hin $\mathrm{zu}$ der einen letztgültigen Darstellung ist. ${ }^{195}$ Erkenntnisfortschritt wird nur erzielt, indem die diversen, immer nur vorläufig gültigen Darstellungen schließlich von der einen Gesamtdarstellung überholt werden.

Wenn Korrespondenz nur bestehen kann, wenn perspektivische Einflüsse ausgeschaltet worden sind, reduziert sich die Rolle des Erkenntnissubjekts auf die eines bloßen Durchlauf- und Abbildungsmediums, dem allenfalls noch gewisse höherstufige interpretatorische Kompetenzen und Aufgaben zugedacht werden. Die Basis des historischen Erkenntnisprozesses bilden auffindbare, man könnte sagen: gegebene Daten, die, werden sie denn unverzerrt aufgenommen, im Erkenntnissubjekt die unfehlbaren Grundlagen, das heißt: sicher gegebene Falsifikations- bzw. Verifikationsinstanzen für weitergehende Interpretationen dieser Daten abgeben. Dabei mag ihre Interpretation einige Unsicherheiten mit sich bringen, wird aber auch sie (methodisch) korrekt durchgeführt, dann besteht auf der interpretatorischen Ebene ebenso eine Korrespondenz zwischen Erkenntnis und Erkenntnisgegenstand wie auf der Datenebene.

Jede Abweichung - und das heißt auch: jede Modifikation und damit jeder Versuch einer Vereinbarung von Standortgebundenheit mit Objektivität - von diesem, mit Popper gesprochen, „Kübelbild“" wird als Anti-Objektivismus, mithin als illegitim und nicht-wissenschaftlich, angesehen. ${ }^{196}$ Wo

195 Vgl. Minks These, dass sich auch die moderne Geschichtsschreibung noch nicht restlos vom Anspruch der Universalgeschichte getrennt habe, eines Tages die diversen Darstellungen der verschiedenen Historiker wenigstens idealiter zu der einen großen Darstellung der einen (universalen) Geschichte aufsummieren zu können (Mink 1987b, 189-195).

196 Vgl. Poppers Kritik an der „Erkenntnistheorie des Alltagsverstandes“ (1993, $61 \mathrm{ff}$.). Der Kern der Erkenntnistheorie des Alltagsverstandes mache das „Kübelbild“ oder auch: die „Kübeltheorie des Geistes“ aus: „Unser Geist ist ein Kübel, anfangs leer, oder mehr oder weniger leer, und in diesen gelangt Material durch unsere Sinne (oder vielleicht durch einen Trichter zur Füllung nach oben), häuft sich an und wird verdaut.“ (Ebd. 61 f.). Die kritische Stoßrichtung ist klar: Diese ,Theorie' geht davon aus, dass unsere Erfahrung das Ergebnis einfachen, unverzerrten Hinschauens und eines daran anschließenden Durchlaufens von (seitens 
der menschliche Erkenntnisapparat aber über seine Rolle als ausschließlich rezeptives Durchlaufgefäß hinausgeht, subjektiviert er die reinen Daten und kompromittiert so die ansonsten durchgängige und objektivitätsverbürgende Korrespondenz vom ersten Sinneseindruck bis zur endgültigen sprachlichen Repräsentation dieses Eindrucks.

Daraus ergibt sich ein methodischer Rigorismus, der davon ausgeht, dass eine strenge Methodik - klassisch findet er in der Geschichtswissenschaft seinen Ausdruck in der historistischen Quellenkritik, wie sie von Ranke propagiert worden ist ${ }^{197}$ - zusammen mit der vollständigen ,Auslöschung“

des Subjekts unveränderten) Daten durch unseren kognitiven Apparat bis hin zu ihrer getreuen sprachlichen Wiedergabe in Form von korrespondierenden Aussagen ist. Der menschliche Wahrnehmungs- und Erkenntnisapparat ist auf eine Rolle als passives Durchlaufgefäß (oder eben: einen Kübel) reduziert. Dabei ist diese Reduktion nicht deskriptiv, sondern normativ zu verstehen: Der menschliche Erkenntnisapparat besitzt die Möglichkeit, subjektive Elemente ins Spiel zu bringen, doch damit verzerrt er die eigentlich objektive Erkenntnis. Die Bezeichnung „Kübelbild“ könnte genügen, um den Verdacht aufkommen zu lassen, es handle sich dabei um einen argumentativen Strohmann, der so nicht vertreten wird oder jemals wurde. Tatsächlich werden sich kaum Autoren finden, die das positivistisch-objektivistische Programm explizit in jedem Punkt befürworten. Und doch sprechen zwei Gründe dafür, die Punkte dieses Programms aufzuführen und sich mit ihnen auseinanderzusetzen: (1) Wenn es sich auch um keine explizit bezogenen Positionen handelt, so kann doch konstatiert werden, dass zumindest stillschweigend ein positivistisches Bild des historischen Erkenntnisgewinns großen Einfluss besitzt. Allenfalls könnte gesagt werden, dass ein solches Bild in genuin geschichtstheoretischen Diskussionen überholt (wenn auch nicht ausgestorben) ist, keinesfalls aber die laufende historische Forschung sich davon freigemacht hätte. (2) Die Argumente gegen perspektivische Geschichtstheorien stützen ihre Plausibilität auf ebendiese vermeintlich überholte Position.

197 Vgl. Vierhaus' Arbeit zu Ranke (Vierhaus 1977). Vgl. auch die historistischen Wurzeln der US-amerikanischen Historiographie Ende des 19., Anfang des 20. Jahrhunderts nach Novick 1988, Kap. 1,I und 1,II, z. B. 39: „If the historian was conscientious, mastered Quellenkritik and the auxiliary sciences, avoided the ,phantoms" of hypotheses, he could produce a body of reliable atomistic facts which ,when justly arranged interpret themselves.' The road to the ultimate history - the definitive, objective, re-creation of the historical past - was open." Vgl. auch Kolmer 2008 für die im 19. Jahrhundert einsetzende positivistische Quellensammlungs- und Editionsarbeit, die aus dem Geist eines umfassenden Sammelns 
der Persönlichkeit des Historikers, also jeglicher subjektiver, parteilicher oder perspektivischer Elemente, ausreicht, um diese objektive Gesamtdarstellung zu erreichen (sofern Quellen vorhanden sind). Die methodologische wie auch persönliche Neutralität des Historikers werden auf die Spuren der Vergangenheit angewandt, wobei das Zusammenspiel dieser Spuren mit der Unparteilichkeit des Historikers und seinem rigorosen Methodenobjektivismus es ermöglicht, die wirklich in der Vergangenheit bestehenden und nicht die von den Quellen fälschlich behaupteten Sachverhalte der Vergangenheit sprachlich abzubilden, ${ }^{198}$ und zwar in dem neutralen Spiegel, als der Sprache angesehen wird. Insgesamt wird versucht, den Objektivitäts- und Wissenschaftlichkeitsgrad der Naturwissenschaften zu erreichen. Deren Objektivität dient als zu erreichender Standard, der durch vorurteilsloses Beobachten, einwandfreie Methoden und unverzerrtes Schildern objektive Darstellungen der Natur hervorbringt. ${ }^{199}$ Kurz, der Wissenschaftler soll ein Spiegel sein, in dem sich klar und unverzerrt die Natur bzw. die Geschichte spiegelt. ${ }^{200}$ Zusammengefasst ergeben die folgenden Grundzüge und Postulate des positivistischen Objektivismus:

(1)Die Vergangenheit existiert(e) (in einem Common-sense-Sinn). (Ontologischer Realismus)

(2)Die Vergangenheit besteht aus vom Betrachter unabhängigen Fakten, die vom Historiker entdeckt oder aufgefunden werden können. (Epistemologischer Realismus)

aller atomisierten Fakten entstanden ist (ebd., 58).

198 Vgl. Mommsen 2000, 46.

199 Eine recht konzise Zusammenfassung dieses Zuges durch einen Soziologen in den 40er-Jahren findet sich bei Appleby et al. 1994 zitiert: ,, The stars have no sentiments, the atoms no anxieties which have to be taken into account. Observation is objective with little effort on the part of the scientist to make it so." (Ebd., 16) In diesem Zusammenhang ist auch das Ideal einer nomologischen Einheitswissenschaft von Bedeutung gewesen. Hier wird auf eine Diskussion dieses positivistischen Zuges verzichtet, weil er nicht wesentlich zum positivistischen Objektivismus gehört, wenn er sich auch historisch mit ihm immer wieder verbündet hat.

200 Vgl. Appleby et al. 1994, 28 f. Wie die ideengeschichtliche Genese dieser Auffassung in der Geschichtswissenschaft aussieht, beschreiben Appleby et al. im zweiten Kapitel von 1994 (52-90). 
(3)Eine vollständige Darstellung dieser Fakten ist möglich. (Universalgeschichtliches Postulat)

(4)Partielle Darstellungen addieren sich zur vollständigen universalgeschichtlichen Darstellung auf. (Aggregationspostulat)

(5)Diese Darstellung soll nicht nur vollständig, sondern auch objektiv sein, das heißt, sie muss dem Dargestellten korrespondieren. Auch partielle Darstellungen der Vergangenheit sind objektiv, sofern sie dem Dargestellten korrespondieren. (Objektivitätspostulat)

(6)Korrespondenz liegt nur vor, wenn keinerlei Perspektivität (oder Subjektivität) in den Erkenntnisprozess oder in die Darstellung der Erkenntnisse eingeflossen ist. (Anti-perspektivisches Korrespondenzpostulat)

(7)Aus (5) und (6) folgt, dass der Historiker unverzerrt die Vergangenheit wiederzugeben hat - seine eigene Perspektive muss, ausgelöscht' werden. (Neutralitätsgebot)

(8)Das Neutralitätsgebot ist nur dann sinnvoll, wenn auch die sprachlichen Darstellungsmittel als transparent aufgefasst werden können. (Sprachliche Transparenz historischer Darstellungen)

(9)Die Tätigkeit des Historikers beschränkt sich darauf, eine etablierte, rigorose Methodik auf die Spuren der Vergangenheit, sprich, die Quellen anzuwenden. Idealiter (d. h. Beachtung des Neutralitätsgebots (7) und ausreichend ausgereifte Methoden vorausgesetzt) gelingt es ihm auf diese Weise, die Tatsachen ans Licht zu bringen, worauf er sie dann - unter erneuter Beachtung von (7) - zu einer objektiven Darstellung im Sinn von (5) und (6) und unter Voraussetzung von (8) zusammenfasst. Die auf diese Weise, entdeckten, aufgefundenen Tatsachen' sind das Fundament jeder weiteren wissenschaftlichen Operation, ihr Prüfstein gewissermaßen. (Historischer Fundamentalismus)

Diese Grundüberzeugungen ergeben die programmatischen Grundzüge des positivistischen Objektivismus, von dem aus er Kritik an, aus seiner Sicht, anti-objektivistischen Geschichtstheorien übt. Mit diesen kritischen Einwänden setzt sich der Abschnitt (4.2) auseinander. Dabei wird nicht nur die Kritik zurückgewiesen, sondern es werden auch wichtige Erkenntnisse 
gewonnen, die im letzten Kapitel in die Grundzüge einer perspektivischen Geschichtstheorie eingefügt werden.

Die kritischen Einwände gegen eine perspektivische Geschichtstheorie stellen aber nicht die einzige Herausforderung des positivistischen Objektivismus an einen Perspektivismus dar. In Abschnitt (4.3) soll exemplarisch der ,positivistische Narrativismus “ untersucht werden. So häufig Positivismus und Narrativität als unvereinbare Gegensätze betrachtet werden, so wenig überzeugend ist diese unterstellte Gegensätzlichkeit über eine gewisse Anfangsplausibilität hinaus. Narrativität und Positivismus können durchaus eine Verbindung eingehen, was für die vorliegende Arbeit von besonderem Interesse ist, weil sich dadurch die Möglichkeit bietet, eine positivistische Theorie zu untersuchen, ohne damit hinter die Position des minimalistischen Narrativismus zurückzufallen, wonach Geschichte wesentlich narrativ ist. 


\subsection{Positivistische Kritik an historischer Standortge- bundenheit}

Ausgehend von der vorgestellten Programmatik argumentiert der positivistische Objektivismus gegen Geschichtstheorien, die eine Vereinbarung von Standortgebundenheit mit Objektivität anstreben. Zunächst wird (in 4.2.1) das universalgeschichtliche Postulat betrachtet werden, das die Grundlage für ein gewichtiges Argument gegen einen historischen Perspektivismus abgibt. Weiter dient das Neutralitätsgebot (7) zusammen mit dem anti-perspektivischen Korrespondenzpostulat (6) und der Transparenz historischer Darstellungen (8) als Grundlage für den immer wieder auftauchenden Relativismusvorwurf (4.2.2). Verwandt mit dem Relativismusvorwurf, wenn auch nicht mit ihm zu verwechseln (was gelegentlich geschieht), ist der Parteilichkeitsvorwurf, der sich ebenfalls aus dem Neutralitätsgebot (7) speist. Ihm wird Abschnitt 4.2.3 gewidmet sein.

\subsubsection{Das universalgeschichtliche Postulat}

Wenn Korrespondenz den begrifflichen Kern des Komponentenmodells ausmacht und korrespondierende Darstellungen nur dann als korrespondierend anerkannt werden, wenn die in ihnen dargestellten Fakten vom Erkenntnissubjekt entdeckt, unverzerrt aufgenommen und neutral zusammengefügt worden sind, dann folgt daraus, dass historische Darstellungen, sofern sie wirklich korrespondieren, sich allmählich zu einer Gesamtdarstellung der Geschichte, einer Universalgeschichte, aufaddieren. Die gegebenen Daten (i. e. „Tatsachen“) stehen, mangels neuer Betrachtungsweisen auf die Quellen, unveränderlich fest. Neue Tatsachen können nicht gefunden werden, es sei denn, es werden bislang unbekannte Quellen entdeckt. Das bedeutet, dass einzelne Darstellungen, soweit sie korrespondieren, beibehalten und zusammen mit anderen korrespondierenden Dar- 
stellungen zu immer umfangreicheren Darstellungen zusammengefügt werden. Sie aggregieren sich oder konvergieren zu der einen Universalgeschichte. Der positivistische Objektivismus erhebt damit einen Vollständigkeits- und einen Konvergenzanspruch dahingehend, dass objektive Erkenntnis (zumindest in the long run) eine vollständige Darstellung der untersuchten Ereignisse, Zustände etc. beinhaltet, die aus verschiedenen objektiven Erkenntnissen (Narrationen) zusammenfließt. ${ }^{201}$

Wenn aber die perspektivische Geschichtstheorie davon ausgeht, dass die Perspektive des Historikers einen unabdingbaren Beitrag zu historischer Erkenntnis liefert und die jeweils eingenommenen Perspektiven nicht immer dieselben gewesen sind noch bleiben werden, sprich, Perspektiven selbst historischem Wandel unterliegen, dann könnte das universalgeschichtliche zusammen mit Aggregationspostulat einen gewichtigen Einwand gegen den Perspektivismus abgeben.

Aus diesem Grund scheint es schwer begreiflich, dass das universalgeschichtliche Postulat und mit ihm sein Vollständigkeits- und Konvergenzanspruch in den wissenschaftlichen Diskussionen keine Beachtung finden. Der Grund hierfür liegt aber auf der Hand. Anders als die Naturwissenschaften, die nach universell und, ewig' gültigen Gesetzen suchen, kann die Geschichte, die immer neue singuläre Ereignisse zum Gegenstand hat, erst dann vollständig dargestellt werden, wenn sie an ihr Ende gekommen ist - was sie im Augenblick aber offensichtlich noch nicht ist. Thesen vom „Ende der Geschichte“ können bestenfalls als metaphorisch oder als publizistische Polemiken betrachtet werden. Während in der Naturwissenschaft neue Individuen lediglich unter universale Gesetze subsumiert werden sollen, ${ }^{202}$ bedeutet der ständige Zuwachs an singulären historischen

201 Dass es sich dabei nicht nur um eine lediglich philosophisch signifikante Gedankenspielerei handelt, sondern um eine Vorstellung, die eng verwandt ist mit der historistisch-positivistischen Grundtendenz am Ende des 19. und Anfang des 20 Jahrhunderts, zeigt Novick 1988, 39 f. Er weist darauf hin, dass die damaligen Vertreter des Historismus in den USA (und nicht nur dort) glaubten, das Ende der Geschichtsforschung sei absehbar, weil alle Dokumente, die vorhanden sind, über kurz oder lang erforscht sein würden und darauf die Darstellung der Geschichte vollständig vorgelegt werden könnte.

202 Diese Darstellung der Naturwissenschaft ist selbstverständlich eine Simplifikati- 
Untersuchungsgegenständen eine prinzipielle Unabgeschlossenheit des historischen Untersuchungsbereiches. In diesem Sinn scheint ein Vollständigkeitsanspruch von vornherein zunichte zu sein. Wozu sich also noch damit aufhalten?

Demgegenüber könnte zu bedenken gegeben werden, dass Vollständigkeit durchaus auch bedeuten könne, ein bestimmtes Ereignis, eine Epoche, das heißt ein historisches Individuum vollständig zu beschreiben, also dem offenen Verlauf der Geschichte insofern Rechnung zu tragen, als das Vergangene allenfalls partiell für vergangen und abgeschlossen erachtet wird, ohne aber insgesamt abgeschlossen zu sein. Auf diese Weise könnte die Darstellung der Ereignisse zumindest dann bis zu einem gewissen Zeitpunkt vollständig sein, wenn sich die Geschichte darauf beschränkte, abgeschlossene, begrenzte historische Individuen zu untersuchen. Dieser Einwand von der partiellen Vollständigkeit historischer Darstellungen $(\mathrm{pV})$ ist - im Gegensatz zur evidenten Unhaltbarkeit der ersten Form des universalgeschichtlichen Postulats - von größerer Bedeutung, denn könnte er plausibilisiert werden, würde dies bedeuten, dass wenigstens eine Form der Konvergenz, die sich auf die Konvergenz aller Darstellungen von bereits restlos abgeschlossenen historischen Ereignissen beschränkt, und mit ihr wenigstens ein begrenzter Vollständigkeitsanspruch gehalten werden kann.

Von überlebenswichtiger Bedeutung für dieses Argument ist die Prämisse, historische Ereignisse könnten als abgeschlossen und begrenzt betrachtet und dargestellt werden. Die Schlacht von Hastings, der Erste Weltkrieg oder die Ermordung Cäsars sind in ihrer zeitlichen Dimension klar umrissen und begrenzt, liegen (mehr oder minder) weit zurück und scheinen als historische Individuen nur auf ihre korrekte, abschließende Beschreibung zu warten, und nur Ereignisse dieser Art (also zum Beispiel nicht die deutsche Wiedervereinigung) sind Teil der partiell vollständigen, weil noch nicht an ihr tatsächliches, unmetaphorisches Ende gekommenen Universalgeschichte. Was zunächst wie eine Binsenwahrheit aussieht - was, wenn nicht ein historisches Ereignis, zumal ein weit in der Vergangenheit liegendes, ist abgeschlossen, begrenzt und in seinen Ausmaßen (exakt) definiert? -, stellt sich genauer angesehen als unplausibel heraus. Drei Gründe spre-

on. 
chen dagegen.

(1) Zunächst kann in Anlehnung an Max Webers Argumentation gegen die Möglichkeit, ein historisches Ereignis vollständig zu beschreiben, darauf verwiesen werden, dass selbst die zeitlich größtmögliche Entfernung eines Ereignisses noch nicht die Möglichkeit ausschließt, dieses Ereignis immer wieder aufs Neue zu beschreiben. Die Ereignisse der Vergangenheit zeichnen sich nach Weber sowohl durch eine intensive als auch eine extensive Unendlichkeit aus. Wer immer eine Darstellung eines historischen Individuums gemäß pV anstrebt, kann diesen Anspruch niemals einlösen, weil er sich der unendlichen Fülle an kognitiv-wissenschaftlich zu beherrschendem ,Material' gegenübersieht, mit dem die Realität nun einmal aufwartet. ${ }^{203}$ Jedes historische Individuum und jede der zu seiner Erklärung vorgebrachten Darstellungen sehen sich einer unendlichen Fülle an Daten gegenüber, die ausschließlich mittels selegierender und abstrahierender $\mathrm{Be}$ griffsbildung in den Griff zu bekommen ist. ${ }^{204}$ Unsere Begriffe, mit deren Hilfe wir versuchen, der ,unendlichen Mannigfaltigkeit“ (Weber) Herr zu werden, sind aspektiv und ,ontologisch' aufgeladen, das heißt, sie sorgen dafür, dass bestimmte Gegenstände als Gegenstände dieser und jener Qualität betrachtet werden, wodurch sie entweder Teil der einen Klasse (Extension) oder einer anderen Klasse von Gegenständen werden. Ähnlichkeit, generische Gleichheit, Vergleichspunkte $u$. a. lassen sich also nur relativ zu bestimmten Begriffsrahmen etablieren, die es mit sich bringen - weil sie eben dem Zweck dienen, den hiatus irrationalis, der zwischen Begriff und

203 Diese These taucht bei Weber immer wieder in WL auf. Sie kann gewissermaßen als ,Axiom‘ der WL gelten. Vgl. Roscher-Aufsatz, 65 ff. und auch OA, 171.

204 Jede historische oder kulturwissenschaftliche Forschung (sofern sie an kausal-genetischen Fragen interessiert ist) ist dazu verurteilt, diesen hiatus irrationalis, der zwischen Realität und ihrer begrifflichen Bearbeitung klafft, zu überbrücken. Zum hiatus irrationalis, der zwischen der unendlichen Mannigfaltigkeit des ,empirisch Gegebenen“ und der begrifflich-kognitiven Erfassung des Empirischen besteht, und dem Zwang zur Abstraktion, der aus diesem hiatus folgt, vgl. Oakes 1988, 19-21. Weber verwendet diesen Begriff eher selten (z. B. aber RoscherAufsatz, 15). Jede empirische Wissenschaft ist diesem hiatus ausgeliefert. Es sei die ,(,negative') Voraussetzung der in jeder empirischen Wissenschaft vollzogenen Stoff-Auslese“" (Roscher-Aufsatz, 75, Anm. 2). 
zu Begreifendem klafft, zu überbrücken -, nur jeweils bestimmte Aspekte einer Realität darstellen zu können und andere dafür unbeachtet lassen zu müssen. Nun sind aber genau diese begrifflichen Mittel nicht ein für allemal festgelegt. Die (extensive wie intensive) Unendlichkeit eines historischen Individuums zwingt den Historiker qua kognitiv endliches Wesen dazu, sein Material in einen Begriffsrahmen zu stellen, der seinerseits historischem Wandel unterliegt. Daher kann, solange Erkenntnis von Begriffsrahmen abhängig ist, nicht einmal $p \mathrm{~V}$ eingelöst werden.

(2) Ein Vertreter von $\mathrm{pV}$ könnte nun aber darauf verweisen, dass es der Wissenschaft ausschließlich auf die Darstellung relevanter, nicht jedoch sämtlicher Sachverhalte ankommt, und dass diese Darstellung partiell vollständig sein kann, weil sie selektiv ist. Sie ist vollständig, wenn alle relevanten Elemente in einer Narration enthalten sind. Relevanz beschränkt die $\mathrm{pV}$ gefährdende Unendlichkeit an narrativ zu integrierenden Ereignissen zumindest im Prinzip, weil ja immerhin denkbar wäre, dass eines Tages sämtliche wissenschaftlich relevanten Begriffsrahmen vorliegen und damit die Möglichkeit gegeben wäre, zumindest die wissenschaftlich relevanten Erkenntnisse vollständig zu erfassen und darzustellen.

PV sieht sich dann aber der Schwierigkeit ausgesetzt, dass das historische Individuum an sich selbst kein Kriterium aufweist, mittels dessen festgelegt werden kann, was einerseits die Beschreibung der Verästelungen des Kontextes (extensive Unendlichkeit - extrinsisch-relationale Eigenschaften) und andererseits den Detailgrad der Beschreibung des historischen Individuums selbst (intensive Unendlichkeit - intrinsische Eigenschaften) limitiert. Es müssen Relevanzkriterien dafür herangezogen werden, was erfasst werden soll. Relevanzkriterien sind jedoch nicht aus den Ereignissen selbst ableitbar, sie müssen vielmehr an die Ereignisse erst angelegt werden. Partielle Vollständigkeit ist damit eine Vollständigkeit relativ zu den Voraussetzungen, Vorgaben und Interessen des Historikers. Diese allerdings unterliegen, wie auch seine Begriffe einem steten Wandel, sind also selbst historisch. Die selektionsfundierenden und -legitimierenden Relevanzkriterien stellen aufgrund ihrer Abhängigkeit von der ihrerseits wandelbaren Gegenwartsposition des Historikers die Auswahl dessen, was als vollständig im Sinn von pV gelten kann, niemals still. Ein Wandel 
in der Betrachtung auch nur der entferntesten historischen Individuen ist die unausweichliche Folge - bis die „Kulturprobleme“ und kulturellen Wandlungen selbst durch das Ende der Geschichte im Literalsinn stillgestellt worden sind.

(3) Eine weitere, auf Danto zurückgehende Überlegung stützt sich auf den wesentlich narrativen Charakter der Geschichte, um zu belegen, dass es vor dem Ende der Geschichte keine Möglichkeit gibt, von auch nur einem einzigen Ereignis zu sagen, welche Rolle es im Verlauf der Geschichte wirklich gespielt habe. Steht aber nicht fest, welche Rolle ein Ereignis in der Geschichte gespielt hat, dann ist es unmöglich, ein Ereignis auch nur partiell vollständig zu beschreiben: (i) Historische Ereignisse sind für sich betrachtet sinnlos oder präziser formuliert: sie sind mit einer unendlichen Fülle an potentiellen ,Sinnkonnexionen' aufgeladen (was Danto wahrscheinlich so nicht gesagt hätte). (ii) Sinnvoll werden sie erst, wenn sie in einen narrativen Zusammenhang integriert werden. (iii) Ein narrativer $\mathrm{Zu}-$ sammenhang verleiht Ereignissen erst dann eine Bedeutung, wenn er abgeschlossen ist, das heißt, wenn die Geschichte (i. S. v. story oder historia rerum gestarum) an ihr Ende gekommen ist. ${ }^{205}$ (iv) Solange aber die Geschichte (i. S. v. res gestae) nicht an ihr Ende gekommen ist, können wir narrative Endpunkte lediglich stipulieren. (v) Daraus folgt - vorausgesetzt, die Geschichte ist noch an keinen Endpunkt gekommen -, dass ausschließlich stipulierte narrative Endpunkte den Fakten einen Sinn verleihen können, der aber jederzeit revidiert werden müsste, wenn die Geschichte (res gestae) eine andere Lesart erzwingt oder ermöglicht, sprich, ein anderes Ende nimmt - und so weiter bis ans Ende der Geschichte. ${ }^{206}$

205 Vgl. Danto 1980, 27 f., Kap. VII, VIII und XI.

206 Zur Illustration führt Danto die narrative Abfolge eines Dramas an. Erst wenn die letzten Zeilen des letzten Aktes gesprochen sind, kann den einzelnen Szenen und Darstellungen wirklich abschließend ein Sinn zugewiesen werden. Solange man nicht weiß, welche dramatische Wendung eine Geschichte nimmt, kann man eine Szene o. ä. nur vorläufig interpretieren. Weberman 1991 geht sogar noch über die Dantosche Argumentation hinaus, indem er behauptet, dass auch die jeweilige Zukunft (oder genauer: die Antizipationen und Prognosen der Zukunft) des Historikers in die narrative Integration mit einfließt. So färbte etwa die Erwartung der atomaren Apokalypse die historische Darstellung des „Kalten Krieges“. (Vgl. We- 
Auch diese Argumentation weist darauf hin, dass die Abgrenzung historischer Individuen nicht einfach gegeben ist, sondern ihrerseits Standards unterliegt, die nicht aus den Quellen oder den Tatsachen allein hervorgehen, sondern sozio-kulturellen und wissenschaftlichen Kriterien unterliegen, die wandelbar sind und daher auch nicht aus sich selbst heraus eine partiell vollständige Darstellung der Geschichte ermöglichen. Die Segmentierung der Geschichte in Ereignisse, Prozesse, Zustände, kurz, historische Individuen als Voraussetzung vollständiger Beschreibbarkeit unterliegt damit also auch gegenstandsexternen Kriterien, die potentiell erst dann festgelegt wären, wenn das Ende der Geschichte eingetroffen wäre, weil nur dieses Ende ausschließen könnte, dass ein früheres Ereignis nicht Teil immer noch einer weiteren Geschichte werden könnte oder seine Rolle anders aufgefasst werden müsste, als dies bisherige narrative Integrationen getan haben. Damit kann nicht einmal eine Aufaddierung eines gewissen Teils historischer Darstellungen plausibel sein, mithin ist weder das universalgeschichtliche noch das Aggregationspostulat eine Gefahr für eine perspektivische Theorie.

Offensichtlich aber ist diese Argumentation problematischer als die beiden ersten. Zu leicht, scheint es, können wir tatsächlich Zäsuren in der Geschichte anlegen. Das Ende des Zweiten Weltkrieges etwa ist leicht zu bestimmen. Auf den ersten Blick mag dieser Einwand berechtigt sein, denn abgeschlossene Ereignisse scheinen sich plausibel als solche kenntlich machen zu lassen. Tatsächlich aber suggeriert dies nur eine vom ,Material vorgegebene Segmentierung, die aber nicht besteht. Zwar kann man etwa das Ende des Zweiten Weltkrieges auf den 08. Mai 1945 legen, die Ereignisketten und Prozesse, die in den Darstellungen des Zweiten Weltkrieges thematisiert werden, sind damit aber nicht zu einem Abschluss gekommen. Viele Entwicklungen und Prozesse, die nach dem Krieg geschehen sind, sind bereits während des Krieges angelaufen. Zäsuren, die innerhalb der Narrationen angesetzt werden, sind alles andere als über jeden Zweifel erhaben. Bestimmte Eigenschaften des historischen Individuums, das abgegrenzt und dann vollständig beschrieben werden soll, sind demnach Teil von Entwicklungen, die vor oder nach den Grenzen eingesetzt haben bzw.

berman 1991, 105-133) 
sich noch fortsetzten. Kontinuitäten und Diskontinuitäten überlagern sich innerhalb eines historischen Individuums. Bestimmte essentielle Elemente der Narration können nur mehr oder weniger willkürlich abgetrennt werden, weil der Narration ein Endpunkt gesetzt werden muss, der dabei kein Endpunkt für alle zur Narration gehörigen Prozesse, Zustände und Ereignisse ist.

Selbst eine vermeintlich noch so einfache Zäsur wie das Ende des Zweiten Weltkrieges setzt voraus, dass das historische Individuum, um das es dem Historiker jeweils zu tun ist, auch genau dieses historische Individuum ist. Wer aber zum Beispiel eine Darstellung des Kampfes zwischen den Kräften der Demokratie und denen des Autoritarismus vorlegen möchte, der kann einige der Ereignisse und Prozesse, die während des Zweiten Weltkrieges stattgefunden haben und als essentieller Bestandteil des historischen Individuums „der Zweite Weltkrieg“ betrachtet werden, ohne irgendeine Rücksicht auf den 8. Mai 1945 völlig unkonventionell narrativ integrieren. Der Waffenstillstand zwischen dem Deutschen Reich und den Alliierten würde nicht als solcher konzeptualisiert und als Endpunkt einer Geschichte betrachtet werden, sondern lediglich als Zwischenstation in einem Ringen, das den Kampf gegen das kaiserliche Japan und die kommunistischen Mächte mitumfasst (und der entsprechend bis zum Jahr 1989 oder noch weiter reichen könnte). Was für die eine Darstellung ein Ende ist, ist für die andere eine Zwischenstation, was für die eine Darstellung ein Fixpunkt ist, ist für die andere ein fließender Prozess etc. Festgelegt sind historische Individuen und ihre jeweiligen narrativen Funktionen nur relativ zu bestimmten Standpunkten, die relative narrative Endpunkte stipulieren und damit nur zu ihnen relative Sinneinheiten konstituieren. Ein historisches Individuum lässt sich nur relativ zu einer gewählten narrativen Perspektive abgrenzen und in seiner historischen Rolle beschreiben.

Die uns zur Verfügung stehenden Möglichkeiten, Sinneinheiten zu bilden, haben also selbst eine Geschichte, stehen ihrerseits also nicht fest und müssen daher immer neue Sinneinheiten hervorbringen - und zwar auch bezüglich längst vertrauter historischer Individuen. Damit werden nicht nur progressiv in der Zeit neue Ereignisse durch neue „Paradigmata“ erfasst, sondern auch alte Ereignisse mit Hilfe neuer „Paradigmata“ interpretiert. 
So ist beispielsweise bezeichnend, welche Anstrengungen auf sozial-ökonomische Verhältnisse in der Geschichtsschreibung des 19. Jahrhunderts verwendet worden sind, als die sog. „Arbeiterfrage“ in aller Munde war (und nachdem Marx und Engels ihren historischen Materialismus formuliert hatten). Die bereits historisch bearbeitete Antike etwa ist erneut auf das sozio-ökonomisch konzeptualisierte Verhältnis von herrschenden Eliten und beherrschten Massen untersucht worden, als die Gegenwart von solchen Fragen beherrscht war. Das bedeutet, dass immer neue sozio-kulturelle Gegenwartsherausforderungen uns immer neue Perspektiven auf die Vergangenheit nehmen lassen und damit immer neue Geschichten zutage fördern werden. PV scheitert demnach an der Historizität der jeweils gegenwärtigen Perspektiven.

\subsubsection{Parteilichkeit vs. Perspektivität}

Das Neutralitätsgebot (7) legt eine weitere positivistische Kritik an historischer Standortgebundenheit nahe. Geschichte perspektivisch schreiben zu wollen, bedeute nichts anderes, als seine eigenen Vorurteile und Voreingenommenheiten geschichtstheoretisch zu legitimieren. Wenn auch die vollständige ,Auslöschung ' der Persönlichkeit des Historikers im Wissenschaftsbetrieb nicht restlos erreichbar sein mag, so handle es sich dabei aber um ein Ideal, das es zu erreichen gilt und das nur zu erreichen ist, wenn geschichtstheoretischen Erschleichungen wie dem perspektivischen Objektivismus ein Riegel vorgeschoben werde. Perspektivität sei Parteilichkeit und müsse daher gleichermaßen abgelehnt werden. ${ }^{207}$ Diese

207 Vgl. Mommsen 1977, 448 und Acham 1977, 394 für die (sinngemäß) gleiche Unterscheidung. Mommsen möchte gegen den Vorwurf des Subjektivismus von seiten des historistischen Positivismus diese Unterscheidung stark machen: „Zwischen Parteilichkeit im Sinne einer Interpretation geschichtlicher Entwicklungen von einem bestimmten Parteistandpunkt aus, die die gewonnenen Ergebnisse zu fundamentalistischen Aussagen hypostasiert, die angeblich aus dem objektiven Geschichtsprozeß resultieren oder doch aus ihm ableitbar sind, und einer Interpre- 
Gleichsetzung wird sowohl von der postmodernen Objektivitätskritik wie vom Positivismus genutzt wenn auch mit jeweils anderen Schlussfolgerungen. Während der Postmodernismus aus der vermeintlichen Unvermeidlichkeit historischer Parteilichkeit die Konsequenz zieht, dass Objektivität unerreichbar ist und daher verabschiedet werden sollte, will der Positivismus dagegen jede Abweichung von seinem Erkenntnisprogramm als Parteilichkeit diskreditieren.

Gegen diesen Vorwurf aber kann der perspektivische Objektivismus sich recht leicht wehren, indem er fundamentale Differenzen zwischen Parteilichkeit und Perspektivität aufzeigt, die sich am besten anhand der Mittel aufweisen lassen, mittels derer der Positivismus Parteilichkeit ausräumen möchte. Wäre nämlich Perspektivität mit Parteilichkeit identisch, würde Perspektivität sich nicht mit den Gegenmaßnahmen gegen Parteilichkeit vertragen. Es wird sich aber zeigen, dass die Remeduren gegen Parteilichkeit einwandfrei mit der These von der Perspektivität historischer Erkenntnis vereinbar ist, weshalb sich Perspektivität von Parteilichkeit frei halten kann und folglich nicht mit ihr identifiziert werden darf.

Generell gesprochen, soll Parteilichkeit durch die Auslöschung der Perspektive (Persönlichkeit) des Historikers beseitigt werden, was genauer gesagt auf drei Wegen geschehen soll: (1) die Korrektur der eigenen parteilich-perspektivisch verzerrten Darstellung durch weitere parteilich-perspektivisch verzerrte Darstellungen; (2) durch die Forderung nach Befolgung eines Ethos der Wissenschaftlichkeit durch den Historiker ${ }^{208}$ und (3)

tation vergangener Wirklichkeit aufgrund einer bestimmten, auf Wertgesichtspunkten und/oder theoretischen Einsichten, die sich der Partialität ihrer selbst bewußt bleibt, ist ein himmelweiter Unterschied.“(Ders. 1977, 448)

208 Bestandteil dieses Ethos ist das, was weiter oben als ,wissenschaftliche Redlichkeit“ bezeichnet worden ist. Ihr wird meist nur wenig Aufmerksamkeit geschenkt. Eine Ausnahme ist Bevir, der die Bedeutung dieser Tugend (,,intellectual honesty") bei der Erreichung objektiver Erkenntnis unterstreicht. Er legt sogar einen Kriterienkatalog vor, der aus der ,intellectual honesty“ hervorgehe und mit dem die Objektivität historischer Interpretationen gesichert werden können (Bevir 1994, 336 f.). Die massive Voraussetzung, auf der seine Argumentation gründet, ist die Annahme, Interpretationen, über deren Wert anhand der genannten Kriterien entschieden wird, seien in Fakten fundiert, über deren Wahrheit in der scientific community Konsens herrsche (ebd., 333 und 337 f.). 
durch die Beziehung zur scientific community ${ }^{209}$.

(1) Wer davon ausgeht, dass die jeweiligen Voraussetzungen, die der Historiker mitbringt, objektiver Erkenntnis im Weg stehen, dessen bevorzugtes Mittel ist es, die verzerrende Wirkung der Persönlichkeit des Historikers weitestgehend auszugleichen, indem weitere, im Grunde ebenso verzerrende Perspektiven in die Darstellung als Korrektiv einbezogen werden sollen, um so (in the long run) die Defizienz perspektivischer Forschung ausgleichen zu können.

Wenn Parteilichkeit nur dann verhindert werden kann, wenn Arbeiten aus anderer Perspektive als Korrektiv für die eigene Erkenntnis herangezogen wird, dann weist der Positivismus damit zwar auf ein wichtiges Mittel hin, wie Parteilichkeit immer wieder zurückgefahren werden kann. Doch damit befindet er sich nur zur Parteilichkeit, nicht aber zur Perspektivität im Gegensatz. Auch der Perspektivismus kann akzeptieren, dass jede Darstellung aus der einen Perspektive auch von Darstellungen aus anderen Perspektiven ergänzt und kritisiert werden muss, ebenso wie die eigene Perspektive von Elementen anderer Perspektiven befruchtet oder korrigiert werden muss.

Worin er sich aber vom Positivismus absetzt, ist die Ansicht, dass das aber nur dazu führen wird, andere Perspektiven unter dem Gesichtspunkt einer weiteren, nämlich der jeweils aktuellen eigenen Perspektive zu betrachten. Wohl kann eine Perspektive modifiziert oder durch eine andere ersetzt, nicht aber aufgehoben oder vollständig suspendiert werden. Der Historiker evaluiert die Erkenntnisse anderer Historiker, muss sie aber von der eigenen Perspektive aus betrachten. ${ }^{210}$ Auf diese Weise ist eine Auslö-

209 Baumgartner macht Parteilichkeit ausschließlich an der Beziehung zur Fachdisziplin fest: „Das Problem der Parteilichkeit in der Historie stellt sich daher in erster Linie als Problem des Verhältnisses von Parteilichkeit und scientific community.“ (Ders. 1977, 433)

210 In diesem Sinn entlarvt Berkhofer 1995 den Versuch, eine vermeintlich parteiliche, weil aus einer Perspektive geschriebenen Geschichte des amerikanischen Mittleren Westens, durch eine polyperspektivische Erzählung zu ersetzen, um damit Perspektivität als solche zu überwinden, als sinnlos. Auch der neue polyperspektivische (multikulturalistische) Ansatz ist seinerseits perspektivisch, er integriert nur andere und vielleicht auch zahlreichere Perspektiven als der eindimen- 
schung der Perspektive gar nicht erst möglich. Perspektivität verträgt sich mit der Kritik durch andere Perspektiven, ohne dass damit Perspektivität als solche hinter sich gelassen werden könnte.

(2) Die eigene Darstellung und die eigene Perspektive kritisch überprüfen zu wollen, bedeutet, von Historikern zu erwarten, dass sie sich als Wissenschaftler durch eine Reihe von Eigenschaften und Handlungsweisen auszeichnen, die essentieller Bestandteil der wissenschaftlichen ,Lebensform' sind. Davon sind am wenigsten kontrovers sicherlich die Forderungen nach der Anerkennung der Regeln der Logik und die Enthaltung von Werturteilen $^{211}$. Teil der wissenschaftlichen Lebensform ist aber auch ein

sionale Ansatz (ebd., 179, 182 und 188). Dabei lässt sich natürlich fragen, wie Perspektiven gezählt werden können. Ist für einen afrikanischen Historiker eine marxistische Geschichte des Imperialismus eine andere Perspektive als eine nationalistische? Sind sie nicht beide aus der Perspektive der weißen ,Unterdrücker“ geschrieben? Und könnte er alle drei Perspektiven integrieren, könnte nicht eine weibliche Historikerin sagen, sie sehe nur die Perspektive von Männern auf die Geschichte des Imperialismus? Offensichtlich ist die Antwort auf die Frage, was eine Perspektive ist, selbst schon wieder von Voraussetzungen des Historikers abhängig.

211 Sich Werturteilen zu enthalten, darf nicht mit einer Reihe von leider immer wieder verwechselten Fragen vermischt werden. Solchen Verwechslungen ist insbesondere Webers sog. ,,Wertfreiheitspostulat" zum Opfer gefallen. Mit Werturteilsfreiheit ist zunächst und am einfachsten gemeint, dass in eine wissenschaftliche Darstellung keine Werturteile hineingehören (vgl. z. B. Weber WA, 497). Davon zu unterscheiden, aber durchaus auch noch in das Wertfreiheitspostulat zu integrieren (vgl. Wissenschaft als Beruf und schon andeutungsweise im Roscher-Aufsatz, 61), ist Webers Ansicht, dass aus den empirischen Erkenntnissen der Kulturwissenschaften keine axiologisch-evaluativen Erkenntnisse folgen. Für sie müssen wir schon selbst sorgen, existenziell-dezisionistisch oder auf anderem Weg. Von diesen beiden Punkten muss wiederum unterschieden werden, dass Weber die Wertabhängigkeit kulturwissenschaftlicher Erkenntnis seiner ganzen Theorie zugrundelegt (vgl. etwa OA passim oder auch WA, 497). Von dieser epistemologischen Voraussetzung Webers können die Putnamsche ,thick ethical concepts“ unterschieden werden, womit er wertgeladene, nur scheinbar rein deskriptive Begriffe bezeichnet (Putnam 2002). Weiter ergibt sich eine fünfte Dimension der Wertfrage, nämlich die nach den Möglichkeiten der (neutralen) empirischen Erfassung von Werten und von moralischer Verantwortung der untersuchten Epochen und Akteure. Letzteres hat Ranke dazu veranlasst, das Amt des Richters von 
gewisser Fallibilismus ${ }^{212}$, mit dem die eigenen Ansichten, Voraussetzungen und Erkenntnisse betrachtet werden sollten, also die Fähigkeit, von den eigenen Voraussetzungen, das heißt Werten, Ansichten, also der eigenen Perspektive insofern abzurücken, als der Historiker immer bereit sein sollte, die eigenen Voraussetzungen auch aufzugeben, sprich, als fallibel anzuerkennen. Er sollte nicht nur, wie im ersten Kapitel bereits angedeutet, vorläufig von der eigenen Perspektive Abstand nehmen und die eigenen Voreingenommenheiten aufgeben können und sich insgesamt wissenschaftlich redlich verhalten, sondern sich darüber hinaus in jeder seiner Voraussetzungen und den auf ihnen basierenden Erkenntnissen als fallibel, das heißt, als potentiell im Irrtum betrachten. Der „Parteimann“ (Baumgartner) unterscheidet sich vom fallibilistischen Historiker dann dadurch, dass er seine Perspektive als sakro-sankt setzt, ohne sich selbst in eine reflektierende Position zu seinen eigenen Voraussetzungen zu bringen. ${ }^{213}$

Die Standortgebundenheit der Geschichtswissenschaft lässt sich aber ausgezeichnet damit vereinbaren, sich auch auf einer Metaebene um Reflexion auf die eigenen Voraussetzungen und um die möglichst gut begründete Ausweisung der eigenen Perspektive zu bemühen. Dabei muss gerade dem Historiker klar sein, dass seine Perspektive selbst ihrerseits eine historische Dimension besitzt und aus diesem Grund immer neue Modifikationen erfahren hat und weiterhin erfahren wird. Dass die Metareflexion zum gut Teil eine der Geschichtswissenschaft externe Leistung ist, ändert nichts daran, dass sie essentiell für die Geschichtsschreibung selbst ist und aus

dem des Historikers zu trennen. Vgl. Mommsen 2000, 48 und 50 für die damit verbundenen Schwierigkeiten, die sich angesichts der Geschichte des 20. Jahrhunderts ergeben haben. Wir haben es folglich mit mindestens fünf verschiedenen Ebenen von Wertfreiheit in den Kulturwissenschaften zu tun, die zumindest analytisch getrennt werden müssen. Daneben ist auch noch die Unterscheidung zwischen context of discovery, in dem Interessen und Emotionen unerlässlich sind, und context of justification, in dem dieselben keine Rolle spielen dürfen, auf die Patzig im Zusammenhang mit der Parteilichkeitsproblematik aufmerksam macht, von großer Bedeutung (ders. 1977, 334).

212 Vgl. Baumgartner 1977 und auch Haskell 1998.

213 Zur eminenten Bedeutung dieser Reflexion auf die eigenen Voraussetzungen vgl. weiter unten Abs. 5.4.2.4. 
diesem Grund in die Betrachtung mit aufgenommen werden muss.

Gerade die Gefahren einer nicht-reflektierten Verabsolutierung der eigenen Perspektive kann in Zeiten, in denen die verschiedenen „Metaerzählungen“ global in Konkurrenz treten, nur noch ethnozentrische Restriktionen mit sich bringen. ${ }^{214}$ Perspektivität erlaubt und fordert die Reflexion auf die eigenen perspektivischen Voraussetzungen, Parteilichkeit dagegen nicht. Perspektivität als solche kann aber nicht hinter sich gelassen werden, weil jede Reflexion auf eine Perspektive nur von einer anderen Perspektive aus erfolgen kann, ebenso wie die Erkenntnis der Historizität der eigenen Perspektive immer nur von einer historisch geformten Perspektive aus gemacht werden kann, die dann ihrerseits wiederum als historisch bedingte erkannt werden muss.

Parteilichkeit kann vermieden werden, indem die eigene Perspektive als fallibel betrachtet und - gute Argumente der konkurrierenden Perspektive vorausgesetzt - auch modifiziert oder aufgegeben wird, nachdem eine reflektierende Position auf die eigenen Voraussetzungen eingenommen worden ist. Nicht vermieden werden kann auf diese Weise aber Perspektivität als solche, denn Perspektiven lassen sich nicht standortunabhängig kritisieren. Sie sind vielmehr in Form einer (Meta-)Kritik von einem anderen Standpunkt aus zu betrachten. Jede Reflexion auf Voraussetzungen kann immer nur von einer anderen Perspektive aus vollzogen werden, was immer nur dazu führen kann, dass eine Perspektive modifiziert oder eine andere angenommen wird - niemals aber dass eine Nicht-Perspektive, ein View from nowhere eingenommen wird, von dem aus Parteilichkeit vermeintlich von vornherein ausgeschlossen werden kann, weil Perspektivität ausgeschlossen werden könne.

(3) Die Haltung des Parteimannes zum methodologischen Element wissenschaftlicher Rationalität führt zu Reglementierung und Beschränkung

214 Vgl. etwa die Verarmung der US-amerikanischen Geschichtsschreibung im 19. Jahrhundert, wie sie von Appleby et al. 1994 konstatiert worden ist. Sie rühre von der Fokussierung auf eine Metaerzählung her, in deren Zentrum der weiße, männliche Farmer stand, der gewissermaßen gottgewollt die Grenzen der amerikanischen Nation immer weiter nach Westen rückte, indem er unternehmerisch, pragmatisch und alle Gefahren verachtend sein Leben aufs Spiel setzte und dabei einen ebenso frommen und wie frugalen Lebensstil pflegte. 
historischer Parteilichkeit durch die scientific community. Teil wissenschaftlicher Rationalität ist es, sich an Anweisungen, Prozeduren, Methoden etc. der jeweiligen Forschungsgemeinschaft zu halten. Dazu gehören die Regeln der Argumentation, der Interpretation, aber auch Regeln für die materielle Basis historischer Untersuchungen oder Regeln der Rechenschaftslegung. Diese Regeln verhindern bis zu einem gewissen Grad, dass der Historiker sich selbst die Geschichte zurechtzulegen vermag, wie er es gemäß seiner Parteilichkeit möchte. Wer von vornherein darauf verzichtet, kritische Quelleneditionen zu benutzen, die der Öffentlichkeit zugänglich sind, wer es versäumt, seine Quellen überhaupt offenzulegen oder wer es unterlässt, seine Forschungen anderen Wissenschaftlern zugänglich zu machen, der macht sich der Parteilichkeit schuldig.

Die Einstellung zu den gängigen und etablierten Praktiken der historischen Fachdisziplin liefert damit ein weiteres Distinktionsmerkmal, wenn auch der Forschungsalltag kaum die notwendige Trennschärfe wird hervorbringen können. Es wird in der Regel schwer festzustellen sein, wo sich ein Historiker gegen eine Methode aus wissenschaftlich-rationalem Bedenken heraus ausspricht oder wo der „Parteimann“ in ihm gewollt seine Augen vor unliebsamen Methoden verschließt. Auch werden Appelle an wissenschaftliche Rationalität erst dann wirksam werden, wenn sie sanktionsbewehrt sind, wenn also die Gemeinschaft der Wissenschaftler (und bis zu eine gewissen Grad auch die interessierten Gruppen in der Gesellschaft) dafür sorgt, dass Verstöße gegen Rationalitäts- und Redlichkeitsforderungen sanktioniert werden. Standards und Sanktionen sind konsensualer Natur. Das bedeutet, dass letztlich Parteilichkeit inhaltlich nur über den Konsens der scientific community festgelegt ist, weil die jeweilige Festlegung dessen, was als parteiliches Verhalten zu betrachten ist, vom Konsens der übrigen Wissenschaftler abhängt. Unparteilichkeit ist daher abhängig von der konsensualen Objektivität. Dabei kann Perspektivität mit jeder dieser konsensualen Festlegungen zurechtkommen, während Parteilichkeit - sollten die Maßnahmen greifen, weil eine wachsame scientific community für ihre Durchsetzung sorgt - auf diese Weise im Zaum gehalten wird.

Auf theoretischer Ebene lässt sich die Trennung zwischen Parteilichkeit und Perspektivität über dieses Distinktionsmerkmal etablieren. Wo der 
„Parteimann“ je nach seiner ideologischen Ausrichtung auf bestimmte Methoden verzichten muss, erlaubt das Bekenntnis zur Perspektivität eine völlige Offenheit gegenüber jeder Methode. Methoden können und müssen ausgesondert werden, was aber nur unter Berufung auf wissenschaftliche Gründe geschehen darf. ${ }^{215}$ Perspektivität ist mit jeder dieser Forderungen vereinbar, denn sie verfügt über das Potential, aus den gleichen Methoden etc. differierende Darstellungen zu gewinnen, ohne sich über die Forderungen nach wissenschaftlicher Rationalität hinweg zu setzen.

Parteilichkeit und Perspektivität unterscheiden sich folglich an entscheidenden Stellen gravierend. Die positivistische Gleichsetzung von allgemein unerwünschter, illegitimer Parteilichkeit mit Perspektivität erweist sich daher als substanzlos.

\subsubsection{Der Relativismusvorwurf}

Eine Vereinbarung von Objektivität mit Standortgebundenheit sei nichts anderes als eine verkappte Form des Relativismus. ${ }^{216}$ Standortabhängige Darstellungen seien nichts anderes als Verstöße gegen das Neutralitätsgebot (7) oder liefen dem Transparenzpostulat (8) zuwider. Der perspektivische Objektivismus sei damit kein Objektivismus mehr, sondern rede einem Relativismus das Wort, weil er von den strengen theoretischen Auflagen

215 Parteilichkeit kann sich aber auch darin manifestieren - wie etwa Mommsen der „orthodoxen“ marxistisch-leninistischen Geschichtsschreibung vorwirft (Mommsen 1977, 448 f.) -, dass der „Parteimann“ sich des Verstoßes gegen das (in diesem Fall: wohlverstandene) „Wertfreiheitspostulat“ Webers schuldig macht: Er leitet aus den empirischen Befunden normative oder axiologische Korollarien ab. Dieser Verstoß gegen das Wertfreiheitspostulat ist allerdings kein Distinktionsmerkmal, das Parteilichkeit von Perspektivität zu trennen vermag, wenn auch der Versuch, die eigene Dogmatik durch Belege aus der Geschichte zu untermauern (was schon immer so war, soll auch so sein etc.), eher einer parteilichen Geschichtsschreibung naheliegen dürfte.

216 Zur Klassifikation des Relativismus und der absolutistischen Gegenpositionen vgl. Harré/Krausz 1996, 23-25, 4-7 und 207 f. 
des positivistischen Objektivismus abrücke. Wer nicht Relativist sein möchte, habe keine andere Alternative, als an den Grundüberzeugungen des positivistischen Objektivismus festzuhalten.

Der Relativismusvorwurf kann aber nur dann als ein gelungener Einwand gegen eine perspektivische Geschichtstheorie anerkannt werden, wenn erwiesen werden kann, dass eine perspektivische Geschichtstheorie einem epistemologisch, gefährlichen' Relativismus gleichzusetzen ist oder epistemologisch ,gefährliche“ Konsequenzen hat. Eine epistemologisch ,gefährliche' Form des Relativismus liegt dann vor, wenn die perspektivischen Voraussetzungen des Erkenntnissubjekts ein objektives Bild des Untersuchungsgegenstandes dadurch verhindern, dass die Kriterien für eine objektives Bild restlos vom Objekt entkoppelt werden. Es wird sich zeigen, dass der Vorwurf des Relativismus, so wie er hier intendiert ist, seinen Zweck jedoch nicht erfüllt, weil die Formen des Relativismus, auf die der positivistische Objektivismus argumentativ beschränkt ist, epistemologisch ungefährlich sind. Gefährliche Formen des Relativismus sind nicht haltbar, daher stehen sie dem Positivismus nicht zur Verfügung.

Die gefährliche Form des Relativismus kann in zwei Untergattungen aufgegliedert werden; zum einen könnte man von individuellem Relativismus, zum anderen von einem Gruppenrelativismus sprechen. Sieht man von immer anzutreffenden epistemischen Restriktionen ab, die jegliche, auch objektive und kompetente Wissenschaftlichkeit limitieren können, kann unter individuellem Relativismus verstanden werden, dass lediglich die individuellen epistemologischen, methodologischen und wissenschaftstheoretischen Standards des jeweiligen Historikers die Kriterien dafür bereitstellen, wann eine historische Darstellung objektiv ist. Wahr oder objektiv ist dann, was diesen individuellen Standards entspricht. Die Geltung der Standards und damit die Objektivität der Erkenntnis liegt in seinem individuellen Ermessensspielraum.

Doch der individuelle Relativismus spielt eine eher untergeordnete Rolle. Allzu idiosynkratische Standards dürften kaum eine Chance haben, sich zu etablieren und a fortiori dürften Erkenntnisse, die diesen Standards gemäß sind, wenig Aussicht darauf haben, allgemein anerkannt zu werden. Da man aber davon ausgehen muss, dass selbst der wissenschaftliche Soli- 
tär Anerkennung für seine Ergebnisse erhalten möchte (geht man davon nicht aus, wäre es für ihn sinnlos, seine Ergebnisse zu veröffentlichen, statt sie in seinem Privatarchiv zu deponieren), kann kein individueller Relativismus, wie er hier von Interesse wäre, plausibel angenommen werden. In der Regel dürfte die besprochene individuelle Voreingenommenheit häufiger anzutreffen sein, als ein genuiner Individualrelativismus. Von größerer Bedeutung für die vorliegende Betrachtung ist daher der Gruppenrelativismus.

Der Gruppenrelativismus taucht seinerseits in zwei Varianten auf: (1) Analog zum individuellen Ermessen bei der wissenschaftlichen Beurteilung historischer Darstellungen kann von einer kollektivem Ermessen gesprochen werden. Weiter oben wurde der fachdisziplinär-konsensualen Objektivität eine zentrale Rolle im Objektivitätsmodell zugedacht. Genauer wurde der scientific community eine Kontrollfunktion zugesprochen, die verhindern sollte, dass der individuelle Forscher idiosynkratische und verfälschende Methoden wählt oder sich über das Unparteilichkeitsgebot hinwegsetzt. Diese Funktion verhindert faktisch, dass das individuelle Ermessen des Historikers allzu abwegige Standards setzt, indem es ,Erkenntnis“, die offensichtlich nur dann als objektiv anerkannt werden kann, wenn ebensolche abwegigen Standards als erkenntnistheoretische Maßstäbe anerkannt werden, die Geltung abspricht und sie damit als nicht mehr weiter diskussionswürdig aus dem wissenschaftlichen Diskurs verbannt.

Es ist ein mehr als geläufiges Phänomen, dass verschiedene wissenschaftliche Denkschulen, das heißt Gemeinschaften von Wissenschaftlern, $\mathrm{zu}$ verschiedenen Ansichten darüber gelangen, was geeignete Methoden und was feststehende Fakten sind und wann ein Forscher parteiisch ist und wann nicht. Die wissenschaftlichen Ergebnisse dieser Gemeinschaften könnten dann nur relativ zu den konsensuell festgelegten Standards als objektiv gelten. Offensichtlich ist ein Teil dessen, was die Perspektive des Historikers ausmacht, genau dieser gruppenspezifische Konsens und damit ein kollektives Ermessen, welches die Korrespondenz der Darstellungen mit der Realität auszuschließen scheint.

So richtig es sein mag, dass verschiedene Denkschulen zu verschiedenen Ergebnissen kommen, so wenig ist damit die Relativismusthese erwiesen. 
In der Vergangenheit hat sich immer wieder gezeigt, dass sich scheinbar unversöhnliche Lager einander annähern oder dass beide von einem anderen Lager integriert werden können oder die Streitpunkte der Diskussion als obsolet und unfruchtbar beiseite gelegt werden mussten. Eine rationale Auseinandersetzung zwischen den Lagern ist immer denkbar, wenn auch nicht immer machbar. ${ }^{217}$ Eine rationale Auseinandersetzung ermöglicht (wenn sie es auch nicht garantiert), dass die gruppenspezifischen Methoden, Fakten und Parteilichkeitsauffassungen einem interkollektiven Rechtfertigungsdruck ausgesetzt sind, ob die jeweils betroffenen Gemeinschaften diese externe ,Einmischung' nun gutheißen (was sie tun sollten, sind sie dem Objektivitätsideal und einem an Wahrheit orientierten Ethos der Wissenschaft wirklich verpflichtet) oder ob sie sich dagegen wehren. Damit ist der jeweilige Konsens der jeweiligen Denkschule immer nur ein vorläufiger und nur ein revidierbarer. Die diskursiv-argumentativen Querverbindungen, seien sie auch noch so kontrovers, sorgen dafür, dass die jeweiligen fachdisziplinären Konsensus nicht inzestuös werden können.

Ein Anhänger des positivistischen Objektivismus wird hier einwenden, dass er mit seiner Relativismusthese nicht meinte, dass eine Gruppe von Historikern absichtlich gegen das Objektivitätsideal verstoßen oder es gar von sich weisen würde, oder dass er nicht glaubt, dass die verschiedenen Denkschulen faktisch einen Raum rationaler Diskussion betreten könnten. Vielmehr möchte er darauf hinweisen, dass der positivistische Objektivismus insofern solchen Phänomenen Rechnung tragen kann, als menschliche

217 Gerade ideologisch oder politisch hart umkämpfte historische Themen stellen eine schier unüberwindliche Anforderung an die Rationalität der beteiligten Historiker (und die sie alimentierenden Institutionen und Interessengruppen). Hier kann es tatsächlich sein, dass eine rationale Auseinandersetzung erst Jahrzehnte, wenn nicht Jahrhunderte nach den einschlägigen Ereignissen stattfinden kann. Es kann sogar unter bestimmten politischen Umständen zur einer regressiven Rationalitätsentwicklung kommen; hier wäre an plötzlich aufflammende ethnische Konflikte o. ä. zu denken, die es für den Zeitraum von Generationen nicht mehr möglich machen, gemeinsam ein rationales Bild einer bislang als gemeinsam aufgefassten und konzipierten Geschichte entstehen zu lassen. Faktisch mögen hier die Hindernisse groß, wo nicht unüberwindlich sein, prinzipiell ist damit nichts ausgeschlossen. 
Erkenntnisfähigkeit faktisch an ihrer Perspektivität scheitern kann, der perspektivische Objektivismus sich dagegen auf eine Sicht festgelegt habe, welche die perspektivische Erkenntnis nicht als Verzerrungen objektiver Erkenntnis betrachtet, sondern als genuin objektive Erkenntnis. Die Folge, so könnte der Einwand weiter lauten, sei eben nicht die, dass ein mühsamer Weg zu wahrhaft objektiver Erkenntnis zurückgelegt werde, sondern die, dass verschiedene Gemeinschaften nur zu verschiedenen Ergebnissen kommen, die untereinander inkommensurabel seien.

Damit liegt die zweite Variante des Gruppenrelativismus vor: (2) Verschiedene Gemeinschaften leben nach verschiedenen Standards, die jeweils objektive Erkenntnis hervorbringen, ohne aber miteinander vergleichbar zu sein, was sie a fortiori keiner rationalen interkollektiven Diskussion mehr zugänglich macht. Es sind aber dann nicht nur Standards, Methoden und Fakten, die inkommensurabel sind, es müssen die Begriffsschemata sein, die dafür sorgen, dass verschiedene Gemeinschaften keinen Zugang zueinander mehr finden, weil ansonsten die Standards einer interkollektiven Diskussion unterworfen werden könnten, was aber gerade ausgeschlossen werden sollte.

Mit dem Begriff der Inkommensurabilität ${ }^{218}$ wird der Gruppenrelativismus subtiler angesetzt als beim ersten Versuch, daher sollten seine Prämissen wenigstens knapp skizziert werden. ${ }^{219}$ (i) Erkenntnis der Welt erfolgt immer begrifflich. Ein zweistufiges Modell, das eine Art von Wahrnehmungsakt mit einem anschließenden, davon aber separierten Konzeptualisierungsakt vorsieht, ist nicht haltbar. Wahrnehmung und begriffliche Erfassung des Wahrgenommenen gehen Hand in Hand. (ii) Konzeptualisie-

218 Vgl. Bevir 1994, 342 f. Seine Argumentation gegen die Inkommensurabilitätsthese ist analog zur hier vorgebrachten. Vgl. auch Hedingers Versuch, die sog. „Subjektivität" der Geschichtswissenschaft mit dem Argument der Inkommensurabilität zu widerlegen (1977, 371 f.). Sein Argumentationsziel besteht allerdings darin, eine weitere, eigenständige Kategorie zwischen „Subjektivität“ und Objektivität aufzumachen, die im Grunde nichts anderes ist als die disziplinäre Objektivität.

219 Vgl. dazu Seebaß 1981, 199-202, wo sich auch weitere Ausdifferenzierungen der Relativismusthese finden; vgl. auch McCullagh 1998, 23 ff. und Davidson 1986; zu verschiedenen Formen des Relativismus Harré/Krausz 1996. 
rung ist abhängig von Begriffsschemata. ${ }^{220}$ Begriffsschemata lenken die Aufmerksamkeit unserer Wahrnehmung auf die für uns relevanten Ausschnitte des Wahrnehmungsfeldes. Sie bestimmen, wie und wann wir wahrnehmen. Unsere Begriffsschemata bestimmen auch, was wir wahrnehmen. (iii) Begriffsschemata sind kulturabhängig. ${ }^{221}$ (iv) Begriffsschemata werden durch Sprachen instantiiert. ${ }^{222}$ Es gibt keinen anderen Zugang zu einem Begriffsschema, als über die Sprache, in der es ausgedrückt wird. (v) Sprachen seien jedoch nicht immer restlos ineinander übersetzbar. (vi) Aus (i) bis (v) folgt: Wenn Sprachen nicht ineinander übersetzbar sind, sie aber der Ausdruck ihrer jeweiligen Begriffsschemata sind und diese wiederum kulturrelativ die Wahrnehmung und Erkenntnis der Angehörigen der jeweiligen Kultur bestimmen, dann verfügen diese Menschen über jeweils wahre Erkenntnis, die jedoch kulturrelativ wahr ist, weil die Maßstäbe für Wahrheit und Falschheit relativ zum jeweiligen Begriffsschema und damit zur jeweiligen Kultur sind. Als kulturrelative Erkenntnis ist sie nicht in ein anderes Begriffsschema, eine andere Sprache oder eine andere Kultur übersetzbar, sie ist inkommensurabel.

Die Anwendung der genannten Schlussfolgerung auf die Geschichtswissenschaft besteht für den positivistischen Objektivismus darin, zu behaupten, dass die perspektivische These von der Objektivität paralleler und immer neuer narrativer Redeskriptionen desselben Gegenstandes eine solche Form des Gruppenrelativismus ist, denn in diesem Zusammenhang von

220 Für eine knappe Explikation von „Begriffsschema“ vgl. Seebaß 1981, 159, Anm. 165 und Abs. 5.3.2 der vorliegenden Arbeit.

221 Beispiele dafür werden in der Literatur zuhauf gegeben: Eskimos nehmen nicht schlicht Schnee wahr, sondern eine uns unbekannte Mannigfaltigkeit an Arten dieser weißen Substanz. Wo ein Medizinmann einen Geist am Werk sieht, sieht der Allgemeinmediziner eine körperliche Erkrankung (und der Psychoanalytiker u. U. eine Neurose), wo der Botaniker Pflanze X der Gattung Y ausmacht, sieht der Laie nur eine Blume auf einer Wiese etc.

222 Das bedeutet nicht, dass hier für eine notwendig sprachliche Form eines Begriffsschemas argumentiert werden soll. Wenn es so etwas wie Begriffsschemata gibt und Aussagen über sie gemacht werden müssen, dann ist es schwer vorstellbar, anders auf sie ,zuzugreifen' als über ihre sprachliche Instantiierung. Vgl. Seebaß 1981, wo gegen die notwendig sprachliche Form der Begriffsschemata argumentiert wird (insb. auf den Seiten 202 und 237-240). 
Wahrheit oder Objektivität zu sprechen, würde bedeuten, jeweils gruppenrelative Kriterien von Objektivität angesetzt zu haben, die aber ihrerseits nicht untereinander verglichen werden könnten, weil sie inkommensurabel seien. ${ }^{223}$ Wer in unterschiedlichen Begriffsschemata wahrnehme und denke, habe auch unterschiedliche und inkommensurable Erkenntnis und damit könne es kein Bild der Welt geben, das Anspruch darauf erheben könnte, das Bild der Welt (i. S. v. objektiv, wahr, korrespondierend) zu sein, also gebe es auch keine gruppenübergreifenden objektiven Darstellungen der Geschichte. Die Standortgebundenheit historischer Erkenntnis würde dann als Grund für die Inkommensurabilität historischer Erkenntnis zu gelten haben, die am deutlichsten wird, wenn Historiker verschiedener Kulturen, dieselben historischen Ereignisse darstellen. Standortgebundenheit habe Inkommensurabilität zur Folge.

Damit dieser Vorwurf stichhaltig sein kann, müsste sich Inkommensurabilität sinnvoll vertreten lassen können. Aus dem Inkommensurabilitätsbegriff ist aber keine plausible Konzeption zu entwickeln, so dass jeder Versuch, aus Standortgebundenheit einen ,gefährlichen“ Relativismus abzuleiten, der sich nicht als bloße Meinungsverschiedenheit zwischen divergierenden Forschungsmeinungen diverser scientific communities, sondern als unüberbrückbare Kluft zwischen Weltsichten entpuppt, daran scheitert, dass aus einer vagen Vorstellung von Inkommensurabilität keine haltbare Position zu gewinnen ist. Ohne eine solche unüberbrückbare Kluft kann dem Perspektivismus aber nichts nachgewiesen werden, was als objektivitätsverhindernd aus seiner Position gefolgert werden könnte.

Aus der Prämisse (iv) - Sprachen seien Instantiierungen der Begriffsschemata - kann das Scheitern der Inkommensurabilitätsthese abgeleitet werden. Es mag zugestanden werden, dass bestimmte Redewendungen und Begriffe mancher Sprachen nicht immer elegant und konzise in andere

223 Die Geschichtswissenschaft wäre - greift man Kuhns Revolutionsmodell wissenschaftlichen Wandels auf - davon gekennzeichnet, dass sie in einem permanenten Revolutionszustand verharren müsste, in dem zwar diverse (inkommensurable) Paradigmata miteinander im Streit lägen, keines davon aber stark genug wäre, die „Revolution“, sprich, den eigenen Durchbruch zur „Normalwissenschaft“ den anderen aufzuoktroyieren. (Vgl. Kuhn 1996, Kap. IX-XIII) 
Sprachen übersetzt werden können. ${ }^{224}$ Daraus folgt jedoch keine prinzipielle Unübersetzbarkeit des semantischen Gehaltes des einen sprachlichen Ausdrucks in Ausdrücke anderer Sprachen. Es mögen gelegentlich langatmige und situationsbezogene Beschreibungen bestimmter Begriffe und Redewendungen erforderlich sein oder auch die semantische ,Teilung' eines fremdsprachigen Begriffs in mehrere Begriffe der Zielsprache notwendig sein. Eine unüberbrückbare Kluft zwischen Fremd- und Zielsprache kann daraus nicht abgeleitet werden. ${ }^{225}$

Die Anforderung an eine Übersetzung Ausdruck für Ausdruck ohne irgendwelche „Reste“ ist viel zu hoch, um akzeptabel zu sein. Kaum eine Sprache dürfte durch eine solch strikte Eins-zu-eins-Übersetzung in eine andere übertragen werden können. Trotz dieses Defizits scheinen sich Kulturen seit frühester Zeit erfolgreich über alles und jedes verständigen zu können. Die Anforderungen sind um des Argumentes willen künstlich hoch angesetzt, wie überhaupt ein Schluss von oberflächliche Ausdrucksdifferenzen in verschiedenen Sprachen auf eine tieferliegende semantische Differenz und damit eine signifikant differierende Erfahrung der Realität kaum überzeugen kann. ${ }^{226}$ Genau diese fundamentale Differenz müsste die

224 Vgl. Harré/Krausz 1996 (47 f.) für einige Beispiele von Übersetzungen, die nicht ohne „Rest“ möglich seien.

225 Vgl. McCullagh 1998, 31 f. für ein von Geertz übernommenes Beispiel einer umfangreichen Übersetzung zweier balinesischer Begriffe in die englische Sprache. Vgl. außerdem Seebaß 1981, 199 für ein knappe Auflistung notorisch schwierig zu übersetzender Begriffe aus diversen Sprachen. Sowohl Seebaß als auch McCullagh betonen die prinzipielle Möglichkeit, auch schwer zu übersetzende sprachliche Äußerungen in eine andere Sprache zu übertragen. Dass dies auf Kosten sprachlicher Eleganz geschieht, dürfte nicht zuletzt ein Grund für die These sein, dass sich Äußerungen in der einen Sprache nur mit Verlusten in Äußerungen der anderen Sprache übersetzen lassen. Daher vermutet Seebaß eine u. a. ästhetisch motivierte Unzufriedenheit hinter entsprechenden Bemerkungen (1981, 203). Auch weitere Phänomene, wie etwa ein gelegentlicher Zwang zur Desambiguierung, werden hier mit hineinspielen.

226 Vgl. Seebaß 1981, Kap. VIII. Seebaß weist auf von ihm unterschiedenen Ebenen (lexikalische, grammatische, etymologische und formal semantische) jeweils nach, dass aus den oberflächlichen Differenzen auf der Ausdrucksseite der Sprache keinesfalls eine tieferliegende semantische Differenz und daran gekoppelt ein 
Inkommensurabilitätsthese aber nachweisen. Dazu reicht es jedoch nicht aus, auf das Scheitern einer Eins-zu-eins-Übersetzung ohne „Rest“ oder auf andere Ausdrucksdifferenzen zu verweisen. Es ist sehr gut möglich, dass der Angehörige einer Kultur eine Pflanze als Dekoration betrachtet, während ein Angehöriger einer anderen Kultur in ihr eine Heilpflanze erkennt. Verständigen können sich beide über ihre Sichtweisen dennoch, sofern einer der beiden die Sprache des anderen gelernt hat. Sie könnten sich selbst dann über ihre, Welten' verständigen, wenn die jeweils andere Seite ihre Beschreibung als absolut gültig voraussetzt, etwa wenn die Pflanze nicht als Dekoration betrachtet werden darf, weil sie als sakro-sankte Heilpflanze solch profanen Betrachtungen nicht unterliegen sollte. Schwierigkeiten in der Übersetzung implizieren keine inkommensurable Erkenntnis der Welt, oder genauer: keinen unüberbückbaren sprach-, begriffsschemaoder kulturrelativen Zugang zur Realität. ${ }^{227}$

Unterstützend kann Davidsons A-priori-Überlegung angeführt werden, ${ }^{228}$ nach der eine vollständige Unübersetzbarkeit keinen Sinn ergibt, während eine partielle Unübersetzbarkeit Inkommensurabilität ausschließt. ${ }^{229}$ Eine partielle Unübersetzbarkeit schließt Unübersetzbarkeit überhaupt aus, weil sich eine Möglichkeit bietet, die vermeintlich unübersetzbaren Aussagen wie Meinungsverschiedenheiten $\mathrm{zu}$ behandeln und das bedeutet: als ver-

differierender kognitiver Zugang zur Realität entspricht, die sich korrespondierend zu den Differenzen der Ausdrucksseite verhalten (ebd., Kap. VIII, 2-4).

„Wenn wir von bloßen Ausdrucksdifferenzen und insignifikanten Begriffsdifferenzen vollständig absehen, bleiben als zweifelsfreie Belege für die Relativitätsthese nur die bekannten Abweichungen bei den Farbwörtern sowie einige streng analoge Fälle, bei denen ein prinzipiell allen Menschen zugängliches Wahrnehmungskontinuum so aufgeteilt wird, daß sich nicht nur verschiedene interne Differenzierungen, sondern echte begriffliche Überlappungen zwischen einzelnen Sprachen ergeben. Nur in diesem vergleichsweise marginalen Bereich und unter gleichzeitiger Anerkennung eines nicht relativen Bezugspunkts ist die Behauptung von den in den verschiedenen Sprachen enthaltenen, unterschiedlichen Arten der, Welterschließung' plausibel, und von signifikanten Unterschieden im ,Denken' kann bislang überhaupt nicht gesprochen werden." (Ebd., 232 f.)

227 Vgl. Seebaß 1981, 228-231, ebenso Murphey 1994, 1-54.

228 Seebaß 1981 deutet auf Seite 233 in Anm. 240 eine ähnliche Überlegung an.

229 Davidson 1986, 281. 
stehbare und verstandene, wenn auch umstrittene Aussagen: Etwas als eine unübersetzbare Aussage aufzufassen, bedeutet, sie bereits verstanden zu haben.

Vollständige Unübersetzbarkeit setze voraus, dass es so etwas wie eine neutrale Position gebe, von der aus Begriffsschemata verglichen werden können. Etwas als inkommensurables Begriffsschema zu erkennen, bedeutet, es im Vergleich zu etwas mit einem anderen Begriffsschema in Beziehung zu setzen. Dieses Etwas (gewissermaßen das tertium comparationis) könnte „ein feststehender Vorrat an Bedeutungen“ sein oder aber eine theorie- und sprachunabhängig zugängliche (und dennoch intersubjektiv vergleichbare) Realität. ${ }^{230}$ Keine der beiden Annahmen ist sonderlich plausibel. Weder kann ein ,Bedeutungshimmel' angenommen werden, der uns dazu verhelfen könnte, absolut unübersetzbare Sprachen miteinander in Beziehung zu setzen, noch können zwei Sprecher sich über die Inkommensurabilität dessen, was sie jeweils als ihre Sprachen ansehen, ,verständigen', indem sie sich eine neutrale, unbeschriebene Realität vor Augen halten und an ihr demonstrieren, dass ihre respektiven Beschreibungen unübersetzbar sind.

Für die hier vorliegende Arbeit bedeutet dies, dass mehrere Darstellungen desselben historischen Individuums aus unterschiedlichen (sozio-kulturell bestimmten) Perspektiven keinesfalls bedeutet, dass ein untragbarer Relativismus zugestanden werden muss. Wie die Begrifflichkeiten und Beschreibungen zwischen Sprachen, wenn auch umständlich und wenig elegant, hin und her übertragbar sind, so sind auch verschiedene narrative Redeskriptionen miteinander vergleichbar und somit a fortiori übersetzbar und das selbst dort, wo eine kulturelle Kluft zwischen den Historikern zu bestehen scheint (also die Gefahr der Inkommensurabilität am größten ist). Erst dort, wo wirklich konträre Narrationen aufeinander treffen, liegt ein wirkliches Problem vor, das jedoch - epistemische Lücken im Beweismaterial einmal außen vor gelassen - nicht unlösbar ist und keinesfalls bedeutet, dass objektive Darstellungen nicht existieren (können). Solche konträren Narrationen sind kein Fall einer Inkommensurabilität von Begriffsschemata oder Perspektiven. Denn wie könnte dieses Verhältnis festgestellt

230 Davidson 1986, 277. 
werden, wenn nicht dadurch, dass die beiden Darstellungen, die als konträr erwiesen werden sollen, verglichen werden sollen? Können sie aber auf ihre Widersprüchlichkeit hin überprüft werden, dann müssen sie übersetzbar sein. Sind sie aber übersetzbar, dann kann das nur bedeuten, dass die in ihnen erhobenen Ansprüche interkollektiv überprüft werden können.

Nimmt man aber für einen Augenblick an, die Inkommensurabilitätsthese ließe sich tatsächlich halten, was hätte der positivistische Objektivist damit gewonnen? Er könnte vielleicht sagen, verschiedene Historikergemeinschaften lebten in verschiedenen Welten (was auch immer damit in einem international eng verzahnten Wissenschaftsbetrieb gemeint sein könnte, sei einmal dahingestellt) und er könnte damit sagen, dass sich die verschiedenen Gruppen nicht mehr untereinander kontrollieren, noch sich gegenseitig unter den beschriebenen Rationalitätsdruck setzen könnten. Doch selbst wenn man dieser Sichtweise um des Arguments willen zustimmte, hätte der positivistische Objektivist noch nicht sein eigentliches Argumentationsziel erreicht, das ja darin besteht, die Kriterien der Objektivität (Wahrheit, Angemessenheit o. ä.) vom Objekt zu entkoppeln. Was aus der Inkommensurabilität folgt, ist die Unmöglichkeit interkollektiver Kontrolle oder des interkollektiven Erkenntnisabgleichs, nicht aber dass die jeweiligen Gruppen keine wahren Aussagen mehr zustande bringen könnten. Denn daraus, dass jede Gruppe einen kulturrelativen begrifflichen Zugang zu den Tatsachen hat und dieser Zugang von Gruppe zu Gruppe ein anderer ist, folgt nicht, dass sie dadurch Tatsachen falsch erkennen und wiedergeben. Es folgt allenfalls - und dies auch nur, wenn man die Prämisse hinzunimmt, dass die kulturrelativen Begriffsschemata nicht dazu geeignet sind, alle Tatsachen in der Welt zu erfassen -, dass keine vollständige Beschreibung der Welt und der Geschichte möglich ist. Das wiederum mag zwar ein Verstoß gegen einen zentralen positivistischen Programmpunkt darstellen, belegt aber dennoch keine prinzipielle Korrespondenzunfähigkeit aufgrund einer Entkopplung kulturrelativer Wahrheitskriterien von der darzustellenden Vergangenheit. Folglich verfehlt der Relativismusvorwurf sein Ziel, jegliche perspektivische Geschichtstheorie als wissenschafts- und erkenntnistheoretisch untragbar darzustellen. 


\subsection{Die geschichtstheoretische Kontaminierungsthese}

Die Versuche, den perspektivischen Objektivismus als prinzipiell anti-objektivistisch zu erweisen, hat der vorige Abschnitt zwar widerlegt, es bleibt dem positivistischen Objektivismus aber die Möglichkeit, sein erkenntnisund wissenschaftstheoretisches Programm so auszubuchstabieren, dass historische Erkenntnisse, wie ein perspektivischer Objektivismus sie für möglich hält, wie eine Kontamination genuin objektiver Erkenntnis aussähen. $\mathrm{Zu}$ diesem Zweck müsste der positivistische Objektivismus ein Substrat auffindbarer Daten ausweisen, die unverzerrt aufgenommen und wiedergegeben werden können.

Ein Versuch, ein solches Substrat zu finden, ist der Versuch des positivistischen Narrativismus, den wesentlich narrativen Charakter der Geschichte für den positivistischen Objektivismus zu vereinnahmen. Dass nicht alle zu einem bestimmten Zeitpunkt faktisch vorliegenden historischen Aussagen perspektivenlose Abspiegelungen vergangener Sachverhalte sind, liegt auf der Hand. Der positivistische Objektivismus müsste den Bereich dessen eingrenzen und ausweisen, was er als Abspiegelung des Datenfundaments betrachten kann und was als von subjektiver und perspektivischer Verzerrung kontaminiert gelten muss.

Wenn objektive historische Erkenntnis in der unverzerrten Abbildung der Vergangenheit besteht, aber nicht alle historischen Aussagen in diesem Sinn objektiv sind, weil sie von verzerrenden Einflüssen „kontaminiert“ sind, dann muss es historische Aussagen geben, die einen bestimmten Bereich historischer Erkenntnis abbilden, der verlässlich abgebildet werden kann und der somit eine verlässliche Basis historischer Erkenntnis liefert, wenn auch die Geschichtsschreibung immer wieder über diesen Bereich hinausgeht, mithin die vorliegenden historischen Darstellungen faktisch von Verzerrungen kontaminiert sind. ${ }^{231}$ Kontaminierung bedeutet dann,

231 Die „Kontaminierungsthese“ in einer abstrakten Form besagt, dass, wenn schon nicht die Wissenschaftler als Individuen und die wissenschaftlichen Institutionen, dann zumindest die Inhalte der Wissenschaft von kultureller Kontaminierung frei sein sollten. (Daston 1998, 21. Dort geht es speziell um die Naturwissenschaften, 
dass bestimmte, potentiell (im positivistischen Sinn:) objektive historische Erkenntnisse von der Perspektive des Historikers kontaminiert sind. Der positivistische Narrativismus bietet einen positiven Vorschlag für eine historiographische Erfassung der Vergangenheit, die vermeintlich frei ist von illegitimer Kontaminierung der Erkenntnis oder es wenigstens sein könnte.

\subsubsection{Der positivistische Narrativismus}

Der positivistische Narrativismus stellt eine Chance dar, das positivistische Programm mit dem historischen Narrativismus in Einklang zu bringen. Er ist innerhalb des Narrativismus eine Unterströmung, die sich von den beiden anderen Versionen des Narrativismus, dem postmodernen und dem minimalistischen, darin unterscheidet, dass er die Korrespondenz zwischen Darstellung und Dargestelltem tatsächlich als eine Abbildungsrelation zwischen der narrativen Repräsentation in der historischen Darstellung und der narrativen Struktur des Dargestellten auffasst. Das Ziel dieses Ansatzes besteht darin, den sogenannten „Impositionalisten“ - etwa L. Mink, H. White und auch F. Ankersmit - das Argument aus den Händen zu winden, die narrativen Strukturen historischer Darstellungen werden im Grunde willkürlich einer Ansammlung von Ereignissen (bzw. Einzelaussagen) auferlegt. Denn dadurch werde, soweit stimmt der positivistische Narrativismus mit der Analyse des postmodernen Narrativismus überein, jedes Korrespondenzverhältnis (und damit die Möglichkeit von wahren Narrationen) ausgeschlossen. Der positivistische Narrativismus (die ,plot-reifiers“ mit Norman) behauptet dagegen, dass historische Darstellungen insofern wahr sind, als sie die narrativen Strukturen der erfahrenen und gelebten Vergangenheit nachvollziehen. ${ }^{232}$

doch die Grundüberzeugung ist auch für die Geschichtswissenschaft zumindest tendenziell dieselbe.)

232 Vgl. Normans Zusammenfassung der Vorteile der Theorie der „plot-reifiers“: „A second virtue of the plot-reifier's account is that it seems to explain how historical narratives can be true. A story about the past is true [...] when it accurately 
Eine solche Auffassung bietet einen Vorteil für den positivistischen Objektivismus. Wenn die These vom wesentlich narrativen Charakter der Geschichte zutrifft, dann wäre es für den positivistischen Objektivismus sicherlich von Vorteil, seinen überkommenen Widerstand gegen die Integration historische Narrativität in die Geschichtstheorie aufzugeben. Ja, er könnte darüber hinaus den narrativen Charakter der historischer Darstellungen geradezu als Chance betrachten, Objektivität in einem simplen Abspiegelungssinn $\mathrm{zu}$ etablieren und damit den wesentlich narrativen Charakter historischer Erkenntnis in den Dienst des positivistischen Verständnisses von Objektivität zu stellen, indem er eine Schicht oder einen Bereich in der Geschichtswissenschaft offenlegt, in der bzw. dem sich ein positivistisches Erkenntnisprogramm, wie es oben skizziert worden ist, potentiell durchführen ließe.

Der positivistische Narrativismus wird als Gegenposition zu einem Narrativismus verstanden, der aus dem narrativen Charakter historischer Darstellungen erkenntnis- und objektivitätsskeptische Schlussfolgerungen zieht, ${ }^{233}$ weil Narrationen keine vorhandenen narrativen Strukturen sprachlich repräsentieren, sondern nachträglich den Ereignissen auferlegt werden. In den oft zitierten Worten Louis Minks: ,[..] to say that the qualities of narrative are transferred to art from life seems a hysteron proteron. Stories are not lived but told. ${ }^{6234}$ In diesem Gegensatz sieht David Carr, der exponierteste Vertreter des positivistischen Narrativismus, sich selbst: „By arguing for the narrative character of human experience, both individual and social, we have been concerned from the start to counter the view of certain theorists [...] that a narrative account is so utterly different in form from the events it portrays that by virtue of this form alone it is constitu-

maps the real narrative structure of the lived past.“"(1998, 158) Welche Schwierigkeiten mit dieser auf den ersten Blick vielleicht noch als plausibel erscheinenden Theorie gerade für die Geschichtswissenschaft entstehen, wird sich noch zeigen. Entsprechend skeptisch ist Norman auch in seiner Einschätzung dieses Vorteils (159).

233 Vgl. die Gegenüberstellung der ,impositionalists“ White und Mink mit dem Ansatz des ,plot-reifiers“ Carr bei Carter 2003, 1 f., Dray 1989b, 131-134 und Norman 1998, 154-156.

234 Mink 1989a, 60 (zweite Hervorh. J. K.). 
tionally condemned to misrepresentation. ${ }^{6235}$ Und Carr weiter über die Gegenseite: „Real events simply do not hang together in a narrative way, and if we treat them as if they did we are untrue to life. Thus not merely for lack of evidence or of verisimilitude, but in virtue of its very form, any narrative account will present us with a distorted picture of the events it relates. ${ }^{\text {"236 }}$

Weil für den positivistischen Narrativismus Geschichtsschreibung ebenfalls wesentlich narrativ ist, die narrative Form der sprachlichen Darstellung dem ,Material' dagegen weder willkürlich auferlegt wird (wie das der postmoderne Narrativismus vorsieht) noch als kontrollierte Konstruktion (wie es der konstruktionistische Narrativismus vorsieht) entsteht, müssen die abzubildenden narrativen Strukturen bereits in der Welt zu finden sein. „The most general point we want to make is that, insofar as such [sc. historical] inquiry results in narrative accounts, these must be regarded not as departure from the structure of the reality they purport to depict, much less a distortion or radical transformation of its character, but as an extension of its very nature. ${ }^{6237}$ „Narrative is not merely a possibly successful way of describing events; its structure inheres in the events themselves. ${ }^{، 238}$

Wo aber sind diese narrativen Strukturen zu finden? Nach Carr ${ }^{239}$ zeichnen sich bereits einfache Handlungen und Wahrnehmungen durch eine Struktur aus, die als proto-narrativ oder narrationsähnlich zu bezeichnen ist. Erfahrungen besitzen notwendig, laut Carr, eine Anfang-Mitte-EndeStruktur, während Handlungen nach dem Zweck-Mittel-Schema strukturiert seien, und das bedeute, sie blickten von einem imaginierten Endpunkt, i. e. dem Zweck, quasi-retrospektiv auf die zu wählenden Mittel und den Zustand vor dem Einsetzen der Handlung als Anfang zurück. Diese Strukturen finden sich auf der Ebene einfacher Handlungen und Wahrnehmungen ebenso wie auf der Ebene komplexer Handlungen, die ihrerseits aus einfacheren Handlungen und Wahrnehmungen, aber auch aus genuinen

235 Carr 1986, 169.

236 Carr 1998, 137.

237 Carr 1986, 169.

238 Carr 1998, 137.

239 Für das Folgende vgl. Carr 1986, Kap. II, IV, VI und ders. 1998. Vgl. außerdem die konzise Zusammenfassung bei Dray 1989b, 135-140. 
Narrationen bestünden. Die narrative Struktur komplexer Handlungen, die sich über lange Zeit erstreckten und daher meist häufig und lange unterbrochen seien, ermöglichten überhaupt erst Handlungen wie das Schreiben eines Buches oder die Erziehung eines Kindes. Darüber hinaus seien aber auch persönliche Selbstauffassungen nichts anderes als narrative Zusammenhänge, die als Narrationen für die jeweilige personale Integrität sorgten.

Doch ist damit die These wirklich belegt, dass narrative Strukturen in der Welt sind? Der positivistische Narrativismus (insbesondere bei Carr, aber auch bei zum Beispiel Carter) schwankt zwischen einer starken ontologisch-epistemologischen und einer schwachen epistemologisch-praktischen These: ${ }^{240}$ Im ersten Fall müsste gezeigt werden können, dass die Welt - und das bedeutet die Ereignisse in der Welt - narrativ strukturiert sind und als solche erfahren und repräsentiert werden; im zweiten Fall wird lediglich gesagt, dass unsere Erfahrung und gleichermaßen unsere Handlungsentwürfe narrativ strukturiert sind, über die Ereignisse selbst wird nichts oder zumindest nicht besonders viel gesagt. (Selbstverständlich wird diese scharfe Dichotomie so nicht von den Vertretern des positivistischen Narrativismus gemacht.)

Die erste, starke Lesart scheint die einzig interessante zu sein, denn nur sie, so hat es den Anschein, kann das Dilemma überbrücken, das sich aus der wesentlich narrativen Form der Geschichtsschreibung und den Ansprüchen des positivistischen Programms ergibt. Doch auch die zweite, schwächere Lesart besitzt dieses Potential, wenn auch in geringerem Maße, weil argumentiert werden könnte, dass man zwar nichts oder nicht viel von der Struktur der Ereignisse wissen kann - außer ihrer temporalen Abfolge und der Tatsache, dass sie irgendwelche Beziehungen untereinander eingehen -, wir aber wissen, dass unsere Erfahrung ebenso wie unsere (individuellen wie kollektiven) Handlungsentwürfe narrativ strukturiert sind - und eben diese Strukturen es sind, die vom Historiker narrativ erfasst werden (müs-

240 Dray (in 1989b, 143 f.) weist auf die Schwierigkeit hin, die sich für eine Einschätzung der Theorie Carrs ergibt, weil Carr die Grenzen zwischen der Frage nach der narrativen Struktur dessen, was erkannt wird, und der narrativen Struktur des Erfahrungsprozesses und der anschließenden narrativen Repräsentation selbst verwischt („,blur“). Vgl. z. B. die Aussagen Carrs in 1986, 46 f. 
sen). Genauso argumentiert Carr, wenn es darum geht, seinen Narrativismus, der sich auf individuelle wie soziale praktische Zusammenhänge konzentriert, auf die Geschichte zu übertragen. Zunächst soll hier die starke ontologisch-epistemologisch Lesart überprüft werden, erst darauf kann die zweite Lesart betrachtet werden.

Im Grunde kann von all den Bereichen, die vermeintlich narrativ strukturiert sein sollen, eigentlich nur der Bereich der Handlungen als narrativ strukturiert gelten. Für die anderen Bereiche gilt lediglich, dass unsere Erfahrung narrativ strukturiert ist bzw. Erfahrung notwendig narrativ sein muss. Die Welt selbst oder die Ereignisse in der Welt sind damit noch lange nicht narrativ strukturiert. Eine wirklich anspruchsvolle metaphysisch-ontologische These, die besagt, dass Ereignisse eine narrative Struktur de rigueur besitzen, wird also gar nicht ernsthaft vertreten.

Es hat den Anschein als sollte die starke (ontologische) These aber gerettet werden, indem die temporale Sequenzierung der Ereignisse in der Welt mit Narrativität identifiziert wird. Doch was sollte damit gewonnen sein? Dass die temporale Sequenzierung von Ereignissen eine Voraussetzung narrativer Erfahrung und narrativer Repräsentation ist, wird kaum von jemandem bestritten. Ebenso wenig wird bestritten, dass Narrationen die Abfolge von Ereignissen nachvollziehen. Damit ist aber noch nicht gesagt, dass Ereignisse selbst narrativ strukturiert sind. Eine bloße Abfolge von Ereignissen ist eben noch keine Geschichte, obschon jede Geschichte voraussetzt, dass es einen Wandel von Ereignisträgern gab und dies wiederum eine temporale Ordnung impliziert. Aus diesem Grund muss eine weitere Prämisse hinzugefügt werden. Die Ereignisse besäßen eine Beginn-MitteEnde-Relation. Wie ist das gemeint? Eine Ereignisveränderung besitzt einen Anfangs- und einen Endzustand und damit eine identifizierbare Mitte. Jedes Ereignis, jede Handlung, jede Wahrnehmung besitzt einen Anfang und ein Ende.

Wie aber kommen die Ereignisse dazu, diese Struktur zu besitzen? Carters Antwort darauf ist verräterisch: Sie werden den Ereignissen von den historischen Akteuren auferlegt. ${ }^{241}$ Ganz im Sinne Carrs sind es die Akteure, die Erfahrungen von Ereignissen repräsentieren, Handlungen entwerfen,

241 Carter 2003, $21 \mathrm{f}$. 
ein Bild von sich selbst haben. ${ }^{242}$ Doch über die Ereignisse selbst ist wieder nicht mehr gesagt, als dass wir sie in bestimmter Weise wahrnehmen, nämlich in einer Beginn-Mitte-Ende-Struktur. ${ }^{243}$ Die gesamte Struktur ist nur eine solche für das Individuum: „They are structures and relations [sc. die der Ereignisse und zwischen den Ereignissen] that exist for the experiencer or the agent in the process of experiencing or acting: they constitute the meaningfulness or direction of the experience or action [...]. ${ }^{\text {‘244 }}$

Vielleicht kann aber eine stillschweigend eingeführte Zusatzprämisse die starke These retten? Wenn unsere Wahrnehmung narrativ strukturiert ist und sie notwendig strukturiert ist, dann bedeutet dies doch nichts anderes, als dass die Ereignisse selbst über ihre temporale Abfolge hinaus, wenigstens irgendeine Struktur besitzen müssen. ${ }^{245}$ Das mag richtig sein und ist gewiss ebenfalls eine der Voraussetzungen des Narrativismus schlechthin, doch reicht irgendeine Struktur für die anspruchsvolle These, dass die Welt

242 Carr 1986, 47: „What counts about the melody as an example of an event is that it is heard as beginning, and each of its phases is heard in anticipation (whether correct or not) of an ending."

243 Carr 1986, 47 und 50 f. Es gibt noch eine Reihe von Variationen der Beginn-Mitte-Ende-Struktur: ,[...] we have found that the notion of ,temporal configuration“ can be elaborated in a number of ways: first as closure or beginning, middle, and end, the most general designation of the phenomenon; then as departure and arrival, departure and return, means and end, suspension and resolution, problem and solution." (Ebd., 49) Diese Varianten zu diskutieren erübrigt sich, weil es an dieser Stelle lediglich um die Frage geht, ob diese Strukturen in der Welt vorhanden sind (im Sinne einer starken ontologischen These) oder ob sie erst vom Individuum hineingelegt werden. Anhand der Spezies der Gattung „Beginn-Mitte-Ende-Struktur" fällt die Antwort noch eindeutiger zugunsten einer nachträglichen subjektiven ,Bearbeitung‘ aus, denn welches Ereignis ist ,an sich` eine Ankunft, oder welche Handlung ,an sich` ein Mittel?

244 Carr 1986, $50 \mathrm{f}$.

245 So zumindest verstehe ich Carr, wenn er sagt: „[...] events are charged with significance they derive from our retentions and protentions. If this is true of our most passive experience, it is all the more true of our active lives, in which we quite explicitly consult past experience, envisage the future, and view the present as a passage between the two. Whatever we encounter within our experience functions as instrument or obstacle to our plans expectations and hopes. Whatever else ,life may be, it is hardly a structureless sequence of isolated events.“ $(1998,141$. 
eine narrative Struktur besitzt, die sprachlich ohne konstruktionistische oder impositionalistische Operationen repräsentiert werden kann, nicht aus. Wieder ist es aber unsere Wahrnehmung, die für die eigentliche Narrativisierung zu sorgen hat. Eine starke ontologische Theorie wird also bestenfalls halbherzig vertreten ${ }^{246}$ - und dürfte auch kaum Chancen haben, plausibel vertreten werden zu können.

Welche Basis für einen Positivismus bildet die schwächere epistemologisch-praktische These? Auch sie besitzt genug Potential für einen positivistischen Objektivismus. Denn es ist immer noch nicht ausgeschlossen, dass eine historische Narration auf den bloßen Nachvollzug vorgegebener narrativer Strukturen festgelegt ist, will sie eine wahre oder objektive Narration sein. Es können nun nur nicht mehr die narrativen Strukturen in der Welt sein, die nachvollzogen werden müssen. Es sind die bereits vorhandenen Narrationen, die sich aus den individuellen und kollektiven narrativen Strukturen individueller Erfahrung, Handlung und personaler Integration und den kollektiver Handlungen und kollektiver Identität ergeben. In diesem Sinn ist zum Beispiel der Startpunkt einer historischen Forschung mit anschließender narrativer Repräsentation die narrative Integration der Gruppe, zu der der Historiker gehört. Es handelt sich also um die narrativen Strukturen, welche die Akteure in ihren Erfahrungen, Handlungsentwürfen und narrativen Selbstwahrnehmungen ,erzählen`. ${ }^{247}$

246 Auffallend, um nicht zu sagen verräterisch, ist Carrs Replik auf den folgenden Einwand: Wenn etwas eine narrative Struktur besitzt oder eine Narration ist, dann braucht es einen Erzähler; wo kein Erzähler, da keine Erzählung. Doch statt diesen Einwand zurückzuweisen oder zu widerlegen, macht ihn Carr sich zu eigen, indem er das jeweilige wahrnehmende und planende Individuum zum Erzähler macht. Das Individuum erzählt sich selbst und anderen die Geschichten, um deren narrative Struktur es geht. Damit allerdings - und darauf weist Dray hin (1989b, $145)$ - ist wieder nichts über die Struktur der Welt gesagt. Schlimmer gar: Wenn Carr ohne Qualifikation dem ,Erzähler-Einwand' zustimmt, dann bedeutet das, dass er auf der Ereignisebene eigentlich gar keine narrative Struktur zulassen kann, ohne entweder dort einen Erzähler einzuführen oder wenigstens auf der Ereignisebene gegen den ,Erzähler-Einwand` zu argumentieren.

247 Aus diesem Grund sei die ,praktische“ Dimension der Narrativität jeder anderen narrativen Dimension vorgängig: „In this sense the narrative activity I am referring to is practical before it becomes cognitive or aesthetic in history and fiction." 
Diese Zusammenhänge seien es, die dem Historiker das Material lieferten, seine Narrationen zu erstellen. ${ }^{248}$, ,[.. ] certainly in a very general way its [sc. the world's] narrative contours are there prior to any particular historian's work and provide the framework within which the latter takes

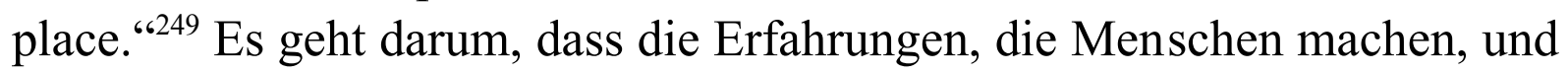
die Handlungen, die sie vollziehen, bereits von uns mit einem narrativen Sinn versehen werden und nicht als sinnlose und unstrukturierte Vorgänge auf uns einstürmen. ${ }^{250}$ Es sind die narrativen Strukturen, mit denen wir Ereignissen und unserer eigenen Existenz einen Sinn verleihen. Genau diese Strukturen müssen dem Historiker als Material dienen. Dabei erkennt Carr an, dass Historiker Geschichte schreiben, indem sie Ereignisse narrativ integrieren und diese Narrationen durchaus auch Elemente enthalten, die als narrative Strukturen nicht im Bewusstsein der historischen Akteure vorhanden waren, aber er insistiert darauf (in einer kryptischen Wendung), dass auch eine solche Narration zumindest an der Form der ursprünglichen Narration teilhaben muss (,their narrative recounting partakes of the form [at least] of the original narrative accounting which is their object ${ }^{6251}$ ). Das bedeutet, wie Carr ein wenig später erläutert, die narrativen Strukturen, die von den Akteuren ,gehabt' werden, sind so etwas wie das Ausgangsmateri$\mathrm{al}^{252}$ (und womöglich auch das Korrektiv) historischer Forschung und Nar-

(Carr 1998, 146)

248 Dabei gibt sich Carr in der Frage nach dem wesentlich narrativen Charakter historischer Darstellungen zunächst agnostisch, betont aber, dass auch deskriptiv-analytische Darstellungen nicht auf narrative Elemente verzichten könnten, wenn es überhaupt vornehmlich deskriptiv-analytische Darstellungen in der Geschichtsschreibung geben könne. Der Grund dafür bestehe darin, dass das Material auch der deskriptiv-analytischen Darstellungen aus den bereits vorhandenen narrativen Strukturen besteht $(1986,175)$.

249 Carr 1986, 175.

250 So zusammenfassend Carr 1998, 145.

251 Carr 1986, $175 \mathrm{f}$.

252 „It may indeed be true that historical research will often penetrate to causal connections [kausale Verhältnisse sind ein Beispiel, das Carr verwendet, um narrative Strukturen zu veranschaulichen, deren sich die historischen Akteure nicht bewusst sind] among events and actions [...] which were hidden from the historical agents themselves. But this is not to deny that these agents lived in a narrative 
rationen.

Die unglückliche Lage, in die sich der positivistische Narrativismus mit einer solchen Theorie gebracht hat, wird nun evident. Auf der einen Seite ist es vollkommen richtig, dass unsere Wahrnehmungen und unsere Handlungen nicht chaotisch auf uns einstürmen respektive nicht von uns chaotisch vollzogen werden, und es mag darüber hinaus sogar richtig sein, dass diese Erfahrungen und Handlungsentwürfe notwendig narrativ sind, ebenso wie personale und kollektive Identität notwendig narrative Mechanismen voraussetzen mag. Auf der anderen Seite ist aber zu fragen, was mit diesen narrativen Strukturen, die in Form erzählter Geschichten in den einzelnen Akteuren aufzufinden sind (oder wenigstens aufgefunden werden können), in der Geschichtswissenschaft angefangen werden soll? Zwei Möglichkeiten sind für die Betrachtungen der vorliegenden Arbeit von Belang.

(1) Diese narrativen Strukturen sind es, die in historischen Darstellungen sprachlich repräsentiert werden müssen. Damit wäre wieder eine einfache Lösung des Objektivitätsproblems erreicht. Sie basiert in diesem Fall nicht mehr auf der starken ontologischen These, dass die Welt narrativ strukturiert ist und wir sie deshalb narrativ strukturiert wahrnehmen und aufgrund dieser isomorphen Korrespondenz auch narrativ repräsentieren können, sondern auf der schwächeren epistemologisch-praktischen These, dass die Ereignisse in der Welt zwar irgendwie strukturiert sind, aber nicht zwangsläufig (oder auch überhaupt nicht) narrativ. Wohl aber sind unsere Erfahrungen, Handlungsentwürfe und personalen wie sozialen Identitäten notwendig durch und durch narrativ strukturiert. Und ebendiese Strukturen nachzuvollziehen, d. h. abzuspiegeln, sei nun die einzige Möglichkeit für den Historiker Korrespondenz und damit Objektivität zu erreichen. Diese Lösung wird zwar vom positivistischen Narrativismus nicht vertreten ${ }^{253}$, es wäre aber an dieser Stelle die einzig konsequente Position, um sich von anderen Positionen überhaupt abgrenzen zu können.

Es mag sein, dass manche Verläufe eine naheliegende Beginn-Mitte-En-

fashion; it is just to say that their story of what they were doing must be revised or indeed replaced by a better one." (Carr 1986, 177)

253 „Thus I am not claiming that the second-order narratives, particularly in history, simply mirror or reproduce the first-oder narratives that constitute their subjectmatter.“(Carr 1998, 151) 
de-Struktur besitzen, aber nicht alle. Auch ist häufig die Narration nicht von Beginn-Mitte-Ende-Strukturen bestimmt, die besonders leicht festzustellen sind. Damit ist aber noch nicht besonders viel über die historische Narration selbst gesagt. Es ist nämlich so, dass, um Zargorins Beispiel aufzugreifen $^{254}$, wir den Beginn und das Ende der Schlacht um Stalingrad doch sehr genau feststellen können. Also habe sie eine solche Beginn-Mitte-Ende-Struktur. Das Problem ist aber, dass historische Narrationen eben nicht mit dem Schlachtbeginn einsetzen und auch nicht mit der Kapitulation der 6. Armee enden. Das mag als marginaler Punkt erscheinen, wirft aber Licht auf eines der zentralen Argumente der ,plot-reifiers“. Selbst wenn bestimmte Veränderungen in der Welt so erfahren werden, wie sie gegeben sind (für den Augenblick sei das einfach einmal so angenommen), das heißt, wie sie als Tatsachen gegeben sind, ist damit noch nicht einfach eine Narrationsstruktur auf der Ebene der sprachlichen Repräsentation gegeben. Die Beginn-Mitte-Ende-Strukturen ,in der Welt' können nicht einfach, i. e. ohne weitere ,logische' Bearbeitung, in sprachliche Repräsentation eins zu eins übertragen werden. Veränderungen von Eigenschaftsträgern und narrative Repräsentation dieser Veränderungen stehen in einem wesentlich komplexeren Verhältnis. So ist es etwa ganz vom Erkenntnisinteresse des Historikers abhängig, wo er seine Narration beginnen lässt. Die eine Narration beginnt vielleicht mit der strategischen Entscheidung Hitlers, die von militärwirtschaftlichen Erwägungen motiviert war, während ein anderer mit der Position der 6. Armee vor ihrem Eingreifen in die Offensive, die zur Schlacht bei Stalingrad geführt hat, beginnt. Der erste ist vielleicht an militärstrategischen Erwägungen interessiert, während es dem anderen um das Schicksal militärischer Großverbände an der Ostfront des Zweiten Weltkrieges zu tun ist. Beide sind sich gewiss über den Anfang und das Ende der Schlacht selbst einig. Doch keiner von beiden lässt seine Narration mit dem Beginn der Schlacht anheben oder mit dem Ende der Schlacht abbrechen.

254 Zargorin 1999, 20 f. Er benutzt dieses Beispiel im Sinn des positivistischen Narrativismus: Der Anfang und das Ende der Schlacht von Stalingrad seien (mehr oder weniger, wie er selbst relativiert) einfach festzustellen und die Wahl, wo die Zäsuren zu machen seien, sei von den Fakten beschränkt. 
Was wird dann aber abgespiegelt, wenn die Narration von Strukturen, von deren Wandlung und von äußeren Zwängen auf die Akteure handelt, derer sich die Akteure nicht bewusst sind? Kann dann immer noch eine Abspiegelung narrativer Strukturen, über welche die historischen Akteure verfügen mussten, sinnvoll vertreten werden? Diese Frage an den positivistischen Narrativismus zu stellen, ist kein überzogenes Ansinnen. Denn das argumentative Ziel des positivistischen Narrativismus besteht darin, Korrespondenz zwischen Darstellung und historischer Vergangenheit gemäß eines einfachen Abspiegelungsstandards aufzufassen. Da historische Darstellungen auch für ihn wesentlich narrativ sind, müssen es vorhandene narrative Strukturen sein, die in einer historischen Darstellung abgespiegelt werden. Ein anderer Weg, etwa über einen ,impositionalism“, soll ja gerade ausgeschlossen werden. Wo können narrative Strukturen vorhanden sein? Diese Frage in einem direkten ontologischen Sinn zu beantworten ist ausgeschlossen worden, es bleibt also nur noch, die narrativen Strukturen ,im Geist' der historischen Akteure zu suchen.

In diesem Fall müsste aber eine enorme Anzahl an historischen Narrationen aus dem Kanon genuiner Geschichtsschreibung entfernt werden. Niemand in der Zeit der (um dieses in diesem Zusammenhang häufig zitierte Beispiel aufzugreifen) „Industriellen Revolution“ war sich langfristiger ökonomischer Entwicklungen und Umbrüche bewusst, als sie sich zugetragen haben. Sollten sich Arbeiten, die sich mit diesen Phänomenen beschäftigen, lediglich auf die vorhandenen zeitgenössischen narrativen Strukturen beschränken? Damit wäre eine nicht tragbare Einschränkung historischer Erkenntnis verbunden. Ganz allgemein gesprochen, wäre dann jeder theoretische Fortschritt in den benachbarten Disziplinen der Geschichtswissenschaft für dieselbe tabu, denn qua Innovation kann diese theoretische Erkenntnis den damaligen Akteuren nicht zur Verfügung gestanden haben, sie können also etwa keine wirtschaftstheoretisch orientierte historische Darstellung der Industriellen Revolution geben. Eine solche wirtschaftstheoretische Darstellung ist aber das Kernstück einer jeden Geschichte der Industriellen Revolution. Eine solche nach heutigen Maßstäben objektive, zentrale und unverzichtbare Auffassung eines historischen Phänomens könnte nicht Teil des historischen Kanons sein, weil die theoretischen Entwicklun- 
gen dem damaligen Zeitgenossen nicht zugänglich waren.

Analog dazu sind bestimmte Narrationen, welche die Entwicklung eines Kollektivs nachvollziehen wollen, nur eingeschränkt denkbar. Eine Reihe wie Die Deutschen und ihre Nation dürfte erst recht spät im Mittelalter einsetzen (und nicht mit dem Ende der sogenannten „Völkerwanderung“), denn kaum ein Bewohner des Frankenreiches dürfte über narrative Strukturen verfügen, in denen das spätere Deutschland auftaucht. ${ }^{255}$ Auch muss klar sein, dass der jeweilige Historiker weiter blicken können muss, als die damaligen Akteure. Es mag durchaus sein, dass tatsächlich eine Narration über die Niederlage einer Armee in einer bestimmten Schlacht ,vorhanden“ ist, und es mag darüber hinaus sogar der Fall sein, dass der Historiker, der sich mit dieser Schlacht auseinandersetzt, keine theoretischen Innovationen oder anachronistischen historischen Individuen verwendet, und dennoch kann er nicht einfach die gegebene Narration schlicht abschreiben, denn womöglich, wurde die Niederlage nicht als eine Folge technologischer Rückständigkeit, die aus struktureller wirtschaftlicher Unterentwicklung resultierte, ,erzählt', sondern als Strafe Gottes für das sündige Verhalten des geistigen Führers. Auch ließe sich kaum eine synthetische Narration rechtfertigen, die aus einer Fülle von Quellen eine nachvollziehbare Geschichte macht. ${ }^{256}$

Die Beispiele für die resultierenden Beschränkungen ließen sich beliebig vermehren, die Richtung dürfte aber klar sein: Eine solche Konzeption, die

255 Vgl. Drays Einwand, dass wir dazu in der Lage sind, Narrationen von Gegenständen zu erzählen, auf die überhaupt keine menschliche Einwirkung stattgefunden hat: die Geschichte der Eiszeit oder die Entstehung eines Gletschers (Dray 1989b, 155). Solche Beispiele aus der Erdgeschichte müssten eigentlich Grund genug sein, den positivistischen Narrativismus als Fundament der Geschichte zu verwerfen. Ihren Wert büßen die Beispiele lediglich deshalb ein, weil selbstverständlich immer noch ein Abgrenzungskriterium der Geschichte gegenüber anderen Wissenschaften ihr anthropologischer Bezug bleibt.

256 Es mag sein, dass für manche ,dunklen Zeitalter', aus denen nur wenige Quellen tradiert sind, ein solcher Ansatz denkbar wäre, was soll aber ein Leser mit den schlicht nacherzählten Berichten z. B. der „Obersten Heeresleitung“ anfangen oder mit den Urkunden der MGH? Wer einmal eine Regestensammlung durchgesehen hat, weiß, wie hilflos er ohne narrativ konstruierten Gesamtzusammenhang dasteht. 
sich lediglich auf den Nachvollzug vorhandener Narrationen stützt, würde Innovation, kritisches Potential und Reichtum der Geschichtswissenschaft so radikal beschneiden oder gar vollständig negieren, dass nichts mehr vom Wissenschaftscharakter der Geschichtswissenschaft übrig bliebe. Diese erste Möglichkeit wird denn auch mit guten Gründen nicht vertreten.

(2) Die zweite Möglichkeit besteht darin, den narrativen Strukturen, von denen die historischen Akteure Kenntnis haben, die sie selbst gebildet oder erfahren haben, als „subject-matter“ (Carr) zu betrachten, von denen aus historische Narrationen ihren Ausgang nehmen, sprich, sie bilden das ,Material', von dem die Geschichtswissenschaft auszugehen hat. Indem sie sich aber von diesen gegebenen narrativen Strukturen abgrenzt, auch wenn sie ihren Anfang von ihnen nimmt, wird sie zu wirklicher Geschichtswissenschaft, zur „,cognitive second-order narrative“.

Zunächst einmal muss dieser Ansatz klarstellen, was er damit eigentlich sagt, denn es ist ein kleiner Trick, mit dessen Hilfe hier unter der Hand zwei Dinge in eins gesetzt werden. Historische Darstellungen schreiben über die Vergangenheit, sie versuchen die Vergangenheit für den Leser darzustellen. Wenn nun die von den Individuen gehabten narrativen Strukturen das Ausgangsmaterial für diese Darstellungen abgeben, dann kann das zweierlei bedeuten ${ }^{257}$ : Entweder diese narrativen Strukturen handeln von etwas, das dargestellt werden soll, das heißt, sie werden als Zeugnisse betrachtet, die etwas über die Vergangenheit aussagen und dieser ausgedrückte Gehalt ist es eigentlich, der das Material der historischen Darstellungen ist. Dann wären es im Grunde nichts anderes als ein komplizierter Weg das bekannte Schlagwort der Historiker ,ad fontes!“ möglichst weitschweifig auszudrücken. Was wäre dann aber die Stellung dieser Quellengattung zu anderen Quellen? Besäßen sie ein epistemologisch-methodologisches Primat? Oder wären schlicht alle Quellen, auch nicht-narrative, dieser Art? Was ist aber mit Sachquellen? Könnten sie nicht mehr als Quellen gelten? Diese Fragen zeigen, dass ein solcher Ansatz an sich kaum haltbar ist. Es zeigt aber auch, dass dann dieser ganze Ansatz keine Besonderheit mehr mit sich bringt, sondern nur noch ein komplizierter Weg ist, Altbekanntes zum besten zu geben.

257 Vgl. Norman 1998, 160, der dort ein ähnliches Argument zu entwickeln scheint. 
Oder aber - und das wäre die zweite Bedeutung dieser Auffassung - das Material der historischen Darstellung sind wirklich die gehabten narrativen Strukturen. Historische Darstellungen sprechen dann tatsächlich nicht mehr über die Vergangenheit, sondern über die narrativen Strukturen, welche die historischen Akteure in ihrem Bewusstsein hatten. Damit allerdings wäre eine grandiose Selbsttäuschung beinahe aller Historiker (außer vielleicht den dekonstruktionistisch orientierten) enttarnt, gehen sie doch davon aus, etwas über die Vergangenheit zu sagen, wenn auch diese Vergangenheit selbst ihnen ausschließlich mittelbar durch die Quellen zugänglich ist. Man mag diese zweite Bedeutung vielleicht noch als groß angelegte Mahnung an den Historiker verstehen, dem Wahrheitsversprechen der Quellen reserviert gegenüber zu treten, aber auch das ist keine wirkliche neue Erkenntnis, sondern methodologischer Standard.

Eine solche Selbsttäuschungskonzeption kann aber auch nicht vertreten werden, denn damit werden natürlich auch sämtliche Formen von Wissen aus ,zweiter Hand' in den Bereich von Selbsttäuschungen gerückt. Ein nicht unerheblicher Teil schon unseres alltäglichen Wissens basiert auf Berichten, Aussagen und Narrationen anderer Menschen. In der Regel gehen wird davon aus (es sei denn, uns liegen Indizien für eine gegenteilige Annahme vor), dass diese Menschen uns schlimmstenfalls falsch informieren (entweder weil sie lügen oder selbst nicht objektiv informiert sind), im günstigsten Fall aber ein mehr oder weniger objektives Bild der Sachverhalte in der Welt geben. Wovon wir nicht ausgehen, ist, dass diese Menschen eigentlich nur über anderer Leute Diskurs sprechen. Wir stehen im Alltag in einer ähnlichen Beziehung zu für uns nicht direkt verifizierbaren Sachverhalten wie der Historiker. ${ }^{258}$ Wenn Meyer seinem Nachbarn

258 An dieser Stelle könnte ein bekanntes skeptisches Argument als Replik verwendet werden: Die Sachverhalte der Vergangenheit sind prinzipiell nicht mehr verifizierbar. Anders als Sachverhalte der Gegenwart, die - zumindest prinzipiell - für alle Zeitgenossen verifizierbar seien, müssen vergangene Sachverhalte als eine besondere Kategorie betrachtet werden. Daher könne die oben genannte Analogie nicht greifen und das Argument sei damit zurückgewiesen. Drei Punkte sprechen dagegen: (1) Was kann hier mit ,prinzipiell“" gemeint sein? Es mag richtig sein, dass es uns prinzipiell verwehrt ist, den Sachverhalt, dass der Zweite Weltkrieg am 1. September 1939 ausbrach, zu verifizieren. Ist es uns aber nicht auch prinzi- 
Schmidt erzählt, er sei als leitender Angestellter in einer Haushaltsabteilung dafür verantwortlich, dass die Lohnzahlungen an die Mitarbeiter pünktlich erfolgen, dann wird Schmidt seiner Frau die Information weitergeben, Meyer sei als leitender Angestellter dafür verantwortlich, dass die Lohnzahlungen pünktlich überwiesen werden. Er bezieht sich auf den Sachverhalt als solchen und gibt ihn als solchen an seine Frau weiter. Schmidts Bezugnahme auf den Sachverhalt (und dessen Weitergabe) ist

piell verwehrt, den Sachverhalt zu verifizieren, dass es jetzt gerade auf dem Mt. Everest schneit? Einem unsportlichen Nicht-Bergsteiger scheinen beide Sachverhalte gleichermaßen unverifizierbar zu sein. Es scheint eine metaphysische Konzeption von Unmöglichkeit unterstellt zu werden, die darauf basiert, dass es nicht möglich ist, in der Zeit zurück zu reisen, wohl aber möglich wäre, ein sportlicher Bergsteiger zu sein, der in diesem Moment auf dem Mt. Everest den Schneefall beobachtet. Vielleicht ist diese Trennung vorstellbar. (Eine mögliche Welt, in der man auf dem Mt. Everest stünde, nachdem man im richtigen Augenblick das Bergsteigen begonnen hätte, ist denkbar, eine mögliche Welt, in der man in der Zeit zurückreist eher nicht, wenn es auch nicht denkunmöglich ist.) Fragwürdig bleibt diese Trennung dennoch. Was aber (2) könnte damit erreicht werden? Faktisch ist ein unsportlicher Nicht-Bergsteiger immer noch darauf angewiesen, dass ihm irgendein Bergsteiger erzählt (oder auf Video zeigt etc.), dass es auf dem Mt. Everest schneit. Er müsste dies entweder im Moment des Schneefalls tun (via „Live-Übertragung“) oder ihm ,historisch“ berichten. Doch dann steht der Zuhörer immer noch in derselben Beziehung zum Schneefall auf dem Mt. Everest wie ein Historiker zum Beginn des Zweiten Weltkrieges - es handelt sich um eine mittelbare Verifikation. Was aber (3) könnte denn prinzipielle unmittelbare Verifikation dieses Sachverhalts für den Einwand gegen die These bringen, dass ein erheblicher Teil unseres nicht-historischen Wissens sich derselben informationellen Genese verdankt wie historisches Wissen? Eigentlich nicht viel, denn es mag sein, dass sich der unsportliche Nicht-Bergsteiger rechtzeitig zum sportlichen 8000erBezwinger trainiert hat, um den Schneefall auf dem Mt. Everest zum fraglichen Zeitpunkt unmittelbar (,mit eigenen Augen“) verifizieren zu können - doch dann kann er leider nicht gleichzeitig das Steigen des Rheinpegels in Köln unmittelbar verifizieren. Prinzipiell mag es uns also möglich sein jeden einzelnen gegenwärtigen Sachverhalt zu verifizieren, es ist uns aber ebenso prinzipiell verwehrt alle gegenwärtigen Sachverhalte unmittelbar zu verifizieren. Die mögliche ,metaphysische' Differenz zwischen der Verifikation eines gegenwärtigen und eines vergangenen Sachverhalts ist demnach völlig unnütz für die Argumentation, selbst wenn sie sinnvoll vertreten werden kann. 
davon unabhängig, ob er ihn von Meyer direkt, von dessen Vorgesetzten oder einer Werbeschrift erfahren hat. Der Zugang zum Sachverhalt kann zum Thema werden, aber in der Regel nur dann, wenn Zweifel am Wahrheitsgehalt der Darstellung aufkommen. Zweifelt Schmidt daran, dass Meyer tatsächlich diese verantwortungsvolle Position innehat, dann wird er seiner Frau nicht sagen, dass $p$, sondern, dass Meyer ihm erzählt habe, dass p.

Doch selbst wenn man von dieser vielleicht $\mathrm{zu}$ sophistisch anmutenden Unterscheidung mit ihren unerfreulichen bzw. unspektakulären Konsequenzen absieht und den positivistischen Narrativismus möglichst entgegenkommend auslegt, bleibt dennoch nichts mehr übrig von der ambitionierten Theorie des positivistischen Narrativismus (entsprechend resümiert Carr auch in einem etwas resignativen Ton, dass es ihm bei seiner Argumentation einzig darauf angekommen sei, nachzuweisen, dass nicht alle narrativen Strukturen nachträglich den Ereignissen auferlegt werden ${ }^{259}$ ), denn er hat sich mit der schwachen epistemologischen Lesart auf eine zum folgenden Entwurf komplementäre Position zurückziehen müssen (wenn er nicht gar auf die Wiederholung des bereits Altbekannten beschränkt bleibt). Denn die Voraussetzungen, die der positivistische Narrativismus macht, sind dieselben, die auch der Konstruktionismus macht: die Veränderung von Eigenschaftsträgern in der Zeit und daraus resultierend eine temporale Ordnung von Ereignissen auf der ontologischen Ebene. Sie ist die Voraussetzung für die wesentlich narrative Erfassung dieser Veränderungen auf der kognitiven Ebene der Erfahrung. Wenn nun auch noch die außerordentliche Autoritätsstellung der gegebenen Narrationen (als lediglich nachzuvollziehende und nachzuerzählende Strukturen) wegfällt, nachdem bereits die starke ontologische These nicht verteidigt werden konnte, dann stellt sich die Frage, welche distinguierende Signifikanz der so hart erkämpften kognitiv-epistemologischen These überhaupt noch zukommt,

$259 \mathrm{Ob}$ ihm das wirklich gelungen ist, bleibt fraglich angesichts der Tatsache, dass die starke ontologische These scheitert, die schwächere epistemologische These aber bestenfalls auch nur zeigen kann, dass unser kognitiver Apparat unerlässliche narrative Strukturen hervorbringt und verwendet, es sich also auch nur um nachträgliche Operationen handelt, wenn auch auf einer der historischen Arbeit vorgängigen Ebene. 
denn auch der konstruktionistische Narrativismus kann sich damit einverstanden erklären, dass die vorhandenen narrativen Strukturen eine herausragende Stellung genießen. Selbstverständlich muss ein Historiker zunächst auch von den narrativen Strukturen in den Quellen ausgehen, und selbstverständlich wird eine gewisse Kontrollfunktion auch von diesen narrativen Strukturen ausgeübt werden. Doch damit ist der perspektivische Charakter der Geschichtsschreibung nicht suspendiert oder gar zurückgewiesen worden, weil an dieser Stelle die wahre historische Arbeit erst beginnt und eben nicht auf den bloßen Nachvollzug und das Wiedererzählen von Narrationen oder narrativen Strukturen beschränkt bleibt. Der positivistische Narrativismus scheitert letztlich daran, dass er sich entweder seiner distinkten Züge beraubt und sich den von ihm bekämpften Positionen bis zur Unkenntlichkeit angleicht, oder aber dass er eine unhaltbare Position vertreten muss. 


\subsubsection{Resümee zum positivistischen Narrativismus}

Das Grundproblem des positivistischen Narrativismus besteht darin, dass er Korrespondenz als Abbildung in einem sehr engen Sinn auslegen möchte. Narrative Strukturen in der Welt (oder nach der schwächeren Lesart: in unserer Erfahrung) korrespondieren narrativen Strukturen in der sprachlichen Repräsentation, andernfalls gebe es keine Korrespondenz. Das aber wäre ein Offenbarungseid für jede Form kognitiver ,Bearbeitung‘ von Informationen gleich welcher Art. Denn warum kann die narrative Form einer historischen Darstellung nicht als kognitives Instrument betrachtet werden, das - analog zu anderen kognitiven Operationen wie deduktiven Schlüssen, inferentiellen Ableitungen, Subsumption unter Begriffe etc. retrospektiv konfigurationale Zusammenhänge ,entdeckt ${ }^{6}$ und sprachlich repräsentiert, ohne damit eine Abbildung voraussetzen zu müssen? Warum sollten gerade Narrationen einer derartig strikten Korrespondenzauffassung unterworfen sein, andere kognitive Instrumente dagegen nicht? Warum zum Beispiel findet sich keine Kritik an Whites (und Minks) Vorstellung einer Chronologie die tatsächlich den Ereignissen korrespondieren kann, warum keine Kritik an Aussagen über kausale Zusammenhänge?260

Die fundamentale Fehleinschätzung der ,plot-reifiers“ liegt also in einer verengten Auffassung von Korrespondenz als strikter Abbildung vorhandener Strukturen. ${ }^{261}$ Könnte die strikte Abbildungsbeziehung glaubhaft vertreten werden, hätte man einen leichten Weg gefunden, sowohl dem wesentlich narrativen Charakter der Geschichtsschreibung Rechnung zu tragen, als auch den Objektivitäts- bzw. Wahrheitsanspruch der Geschichtswissen-

260 Vgl. Dray 1989b, 152. In all diesen und auch anderen Formen sprachlicher Repräsentation scheint die Forderung nach einer derart strikten Strukturgleichheit nicht notwendig zu sein. Warum muss nur die narrative Struktur als „,natürlich“, d. h. als nicht retrospektiv, auferlegt oder in irgendeiner Form Teil einer nachträglichen ,logischen' Operation angenommen werden, andere Formen sprachlicher Repräsentation von Zusammenhängen dagegen als logisch-retrospektive Aufbereitungen von in solcher Form nicht aufzufindenden Zusammenhängen - Formen, die permanent Verwendung in Narrationen finden?

261 Vgl. auch Norman 1998, 159 zu diesem Punkt. 
schaft einlösen zu können.

Leider stellt genau diese strikte Auffassung von Korrespondenz den geeigneten Angriffspunkt für die objektivitätsskeptischen Argumente des postmodernen Narrativismus dar. Dieser wiederum verwirft die Möglichkeit von Wahrheit und Objektivität, weil er plausibel zu zeigen vermag, dass eine solch strikte Abbildungsbeziehung unhaltbar ist. Dabei scheint er, auf den ersten Blick zumindest, dem variablen Charakter der Geschichtsschreibung gerecht zu werden, der es etwa erlaubt, theoretische Neuerungen in die historische Darstellung mit einfließen zu lassen, was der positivistische Narrativismus jedoch ausschließen muss, möchte er seinen distinkten Charakter nicht verlieren. Wird Korrespondenz in einem strikten Abbildungssinn verstanden, dann kann die variable Leistungsfähigkeit der Geschichtsschreibung nicht mehr erklärt werden, also bleibt nur der Impositionalismus, i. e. der postmoderne Narrativismus, als akzeptable Lösung. Der postmoderne Narrativismus verlangt nun aber seinerseits ein Opfer: die Möglichkeit objektiver historischer Darstellungen muss aufgegeben werden.

Keine der beiden betrachteten Herangehensweisen an den Widerstreit zwischen Standortgebundenheit und Objektivität, ob nun die postmodernnarrative oder die positivistisch-objektive, konnte aber die eigene Position stark machen resp. die Vereinbarkeit von Standortgebundenheit und Objektivität überzeugend widerlegen. Der Weg ist nun also frei für eine Position, die zwischen den Extremen liegt. 